\title{
Large-scale Unit Commitment under uncertainty
}

\author{
M. Tahanan - W. van Ackooij • A. Frangioni • \\ F. Lacalandra
}

the date of receipt and acceptance should be inserted later

\begin{abstract}
The Unit Commitment problem in energy management aims at finding the optimal productions schedule of a set of generation units while meeting various system-wide constraints. It has always been a large-scale, non-convex difficult problem, especially in view of the fact that operational requirements imply that it has to be solved in an unreasonably small time for its size. Recently, the ever increasing capacity for renewable generation has strongly increased the level of uncertainty in the system, making the (ideal) Unit Commitment model a large-scale, non-convex, uncertain (stochastic, robust, chance-constrained) program. We provide a survey of the literature on methods for the Uncertain Unit Commitment problem, in all its variants. We start with a review of the main contributions on solution methods for the deterministic versions of the problem, focusing on those based on mathematical programming techniques that are more relevant for the uncertain versions of the problem. We then present and categorize the approaches to the latter, also providing entry points to the relevant literature on optimization under uncertainty.
\end{abstract}

Keywords Unit Commitment · Uncertainty · Large-Scale Optimization · Survey

\section{Milad Tahanan}

Ecole Centrale Paris, Grande Voie des Vignes, Chatenay-Malabry, France, E-mail: miladtahanan@gmail.com

Wim van Ackooij

EDF R\&D. OSIRIS, Avenue du Général de Gaulle 1, F-92141 Clamart Cedex, France, E-mail: wim.van-ackooij@edf.fr

Antonio Frangioni

Dipartimento di Informatica, Università di Pisa, Largo B.Pontecorvo 3, 56127 Pisa, Italia, E-mail: frangio@di.unipi.it

Fabrizio Lacalandra

QuanTek S.r.L, Via G. Marconi, 3, 40122 Bologna, Italia, E-mail: fabrizio.lacalandra@quantek.it

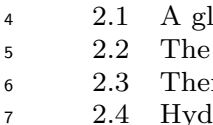


3.2 .3 Recent trends in MILP techniques . . . . . . . . . . . . . . . . . . . . . . . . . . 15

3.3 Lagrangian and Benders Decomposition

3.4 Augmented Lagrangian Relaxation . . . . . . . . . . . . . . . . . . . . . . . . . . . . . . . . . . . . . . . 19

3.5 (Meta-)Heuristics . . . . . . . . . . . . . . . . . . . . . . . . . . . . . . 19

3.5.1 Operator rule based: Priority Listing . . . . . . . . . . . . . . . . . . . . . . . . . 19

3.5 .2 Guided Random Exploration _ . . . . . . . . . . . . . . . . . . . . . . . . . . . . . . . . . 20

4 Methods for the Uncertain Unit Commitment . . . . . . . . . . . . . . . . . . . . . . . . . . . . . . 20

4.1 Dealing with Uncertainty in UC . . . . . . . . . . . . . . . . . . . . . . . . . . . . 21

4.1 .1 Dealing with uncertainty in the model . . . . . . . . . . . . . . . . . . . . . . . . . 21

4.1.1.1 Stochastic optimization. . . . . . . . . . . . . . . . . . . . . . . . . . . 21

4.1.1.2 Robust optimization. . . . . . . . . . . . . . . . . . . . . . . . . . . . 22

4.1.1.3 Chance-Constrained Optimization. . . . . . . . . . . . . . . . . . . . . . . . . . . 22

4.1.1.4 The link between RO and CCO. . . . . . . . . . . . . . . . . . . . . . . 22

4.1 .2 Modelling and solution choices . . . . . . . . . . . . . . . . . . . . . . . . . . . . 22

4.1.2.1 The choice of recourse decisions. . . . . . . . . . . . . . . . . . . . . . . . . . . . . . . . . 22

4.1.2.2 Direct approaches vs. decomposition. . . . . . . . . . . . . . . . . 23

4.2 Stochastic Optimization (Scenario-Tree) approaches . . . . . . . . . . . . . . . . . . . . . . 23

4.2 .1 Mixed Integer Linear Programming . . . . . . . . . . . . . . . . . . . . . . . . . . . . . . . . 24

$4.2 .2 \quad$ Scenario Decomposition $\ldots \ldots \ldots \ldots$. . . . . . . . . . . . . . . . . . . . . . . . 24

$4.2 .3 \quad$ Unit (Stochastic) Decomposition . . . . . . . . . . . . . . . . . . . . . . . . . . . . . . 25

4.2 .4 Benders(-Like) Decomposition . . . . . . . . . . . . . . . . . . . . . . . . . . . . . . 26

4.3 Robust Optimization approaches . . . . . . . . . . . . . . . . . . . . . . . . . . 26

4.4 Chance-Constrained Optimization approaches . . . . . . . . . . . . . . . . . . . . . . . . . . . . . . 27

5 Concluding Remarks . . . . . . . . . . . . . . . . . . . . . . . . . . . . . . . . . 29

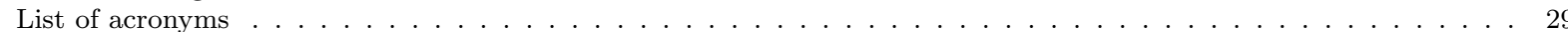

\section{Introduction}

In electrical energy production and distribution systems, an important problem deals with computing the production schedule of the available generating units in order to meet their technical and operational constraints and to satisfy some system-wide constraints, e.g., global equilibrium between energy production and energy demand. The constraints of the units are very complex; for instance, some units may require up to 24 hours to start. Therefore, such a schedule must be computed (well) in advance of real time. The resulting family of models is usually referred to as the Unit Commitment problem (UC), and its practical importance is clearly proven by the enormous amount of scientific literature devoted to its solution in the last four decades and more. Besides the very substantial practical and economical impact of UC, this proliferation of research is motivated by at least two independent factors:

1. on the one hand, progress in optimization methods, which provides novel methodological approaches and improves the performances of existing ones, thereby allowing to tackle previously unsolvable problems;

2. on the other hand, the large variety of different versions of UC corresponding to the disparate characteristics of electrical systems worldwide (free market vs. centralized, vast range of production units due to hydro/thermal/nuclear sources, ....).

Despite all of this research, UC still cannot be considered a "well-solved" problem. This is partly due to the need of continuously adapting to the ever-changing demands of practical operational environments, in turn caused by technological and regulatory changes which significantly alter the characteristics of the problem to be solved. Furthermore, UC is a large-scale, non-convex optimization problem that, due to the operational requirements, has to be solved in an "unreasonably" small time. Finally, as methodological and technological advances make previous versions of UC more accessible, practitioners have a chance to challenge the (very significant) simplifications that have traditionally been made, for purely computational reasons, about the actual behavior of generating units. This leads to the development of models incorporating considerable more detail than in the past, which can significantly stretch the capabilities of current solution methods.

A particularly relevant recent trend in electrical systems is the ever increasing use of intermittent (renewable) production sources such as wind and solar power. This has significantly increased the underlying 
uncertainty in the system, previously almost completely due to variation of users' demand (which could however be forecast quite effectively) and occurrence of faults (which was traditionally taken into account by requiring some amount of spinning reserve). Ignoring such a substantial increase in uncertainty levels w.r.t. the common existing models incurs an unacceptable risk that the computed production schedules be significantly more costly than anticipated, or even infeasible (e.g., 205). However, incorporating the uncertainty in the models is very challenging, in particular in view of the difficulty of the deterministic versions of UC.

Fortunately, optimization methods capable of dealing with uncertainty have been a very active area of research in the last decade, and several of these developments can be applied, and have been applied, to the UC problem. This paper aims at providing a survey of approaches for the Uncertain UC problem (UUC). To the best of our knowledge no such survey exists, while the literature is rapidly growing. This is easily explained, besides by the practical significance of UUC, by the combination of two factors: on one hand the diversity of operational environments that need to be considered, and on the other hand by the fact that the multitude of applicable solution techniques already available to the UC (here and in the following we mean the deterministic version when UUC is not explicitly mentioned) is further compounded by the need of deciding how uncertainty is modeled. Indeed, the literature offers at least three approaches that have substantially different practical and computational requirements: Stochastic Optimization (SO), Robust Optimization (RO), and Chance-Constrained Optimization (CCO). This modeling choice has vast implications on the actual form of UUC, its potential robustness in the face of uncertainty, the (expected) cost of the computed production schedules and the computational cost of determining them. Hence, UUC is even less "well-solved" than UC, and a thriving area of research. Therefore, a survey about it is both timely and appropriate.

We start with a review of the main recent contributions on solution methods for UC that have an impact on those for the uncertain version. This is necessary, as the last broad UC survey 290 dates back some 10 years, and is essentially an update of [349]; neither of these consider UUC in a separate way as we do. The more recent survey [127] provides some complements to 290] but it does not comprehensively cover methods based on mathematical programming techniques, besides not considering the uncertain variants. The very recent survey [337 focuses mainly on nature-inspired or evolutionary computing approaches, most often applied to simple 10-units systems which can nowadays be solved optimally in split seconds with general-purpose techniques; furthermore these methods do not provide qualified bounds (e.g., optimality gap) that are most often required when applying $\mathrm{SO}, \mathrm{RO}$ or $\mathrm{CCO}$ techniques to the solution of UUC. This, together with the significant improvement of solving capabilities of methods based on mathematical programming techniques (e.g., Lagrangian or Benders' decomposition methods, Mixed Integer Linear Programming approaches, ...), justifies why in the UC-part of our survey we mostly focus on the latter rather than on heuristic approaches.

Because the paper surveys such a large variety of material, we provide two different reading maps to the readers:

1. The first is the standard reading order of the paper, synthesized in the Table of Contents above. In Section 2 we describe the varied technical and operational constraints in (U)UC models which give rise to many different variants of UC problems. In Section 3 we provide an overview of methods that deal with the deterministic UC, focusing in particular onto methods dealing with large-scale systems and/or that can be naturally extended to UUC, at least as subproblems. In particular, in $\$ 3.1$ we discuss Dynamic Programming approaches, in 3.2 we discuss Integer and Mixed Integer Linear Programming (MILP) approaches, while in $\$ 3.3$ and $\$ 3.4$ we discuss decomposition approaches (Lagrangian, Benders' and Augmented Lagrangian), and finally in $\$ 3.5$ we (quickly) discuss (Meta)Heuristics. UUC is then the subject of Section 4 in particular, 4.2 presents Stochastic Optimization (Scenario-Tree) approaches, 4.3 presents Robust Optimization approaches, and 84.4 presents Chance-Constrained Optimization approaches. We end the paper with some concluding remarks in $\$ 5$, and with a list of the most used acronyms.

2. The second map is centered on the different algorithmic approaches that have been used to solve (U)UC. The main ones considered in this review are: 
- Dynamic Programming approaches, which can be found in $3.1,33.2 .2$, 3.3 , $3.5 .2, \$ 4.1 .1 .1$ $\$ 4.2 .1$, \$4.2.3, \$4.2.4 and \$4.4

- Mixed-Integer Programming approaches, which can be found in $\$ 3.2,33.3, \$ 4.1 .2 .2, \$ 4.2, \$ 4.2 .1$ $\$ 4.2 .3$, \$4.2.4, 4.3 , and 4.4

- Lagrangian Relaxation (decomposition) approaches, which can be found in $\$ 3.2 .2$ \$3.3, 3.5 .2 . $\$ 4.2 .1, \$ 4.2 .2,44.2 .3$, \$4.2.4, and $\$ 4.4$

- Benders' decomposition approaches, which can be found in \$3.2.2, \$3.3, \$4.2, \$4.2.1, \$4.2.2, \$4.2.3 $\$ 4.2 .4$, and $\$ 4.3$.

- Augmented Lagrangian approaches, which can be found in $\$ 3.3$, $\$ 3.4$, and $\$ 4.4$.

- other forms of heuristic approaches, which can be found in $\$ 3.1, \$ 3.2 .2, \$ 3.3, \$ 3.5, \$ 4.1 .2 .1, \$ 4.2 .2$, and 4.2 .3 .

\section{Ingredients of the Unit Commitment problem}

We start our presentation with a very short description of the general structure of electrical systems, presenting the different decision-makers who may find themselves in the need of solving (U)UC problems and their interactions. This discussion will clarify which of the several possible views and needs we will cover; the reader with previous experience in this area can skip to $\$ 2.1$ for a more detailed presentation of the various ingredients of the (U)UC model, or even to $\$ 3$ for the start of the discussion about algorithmic approaches.

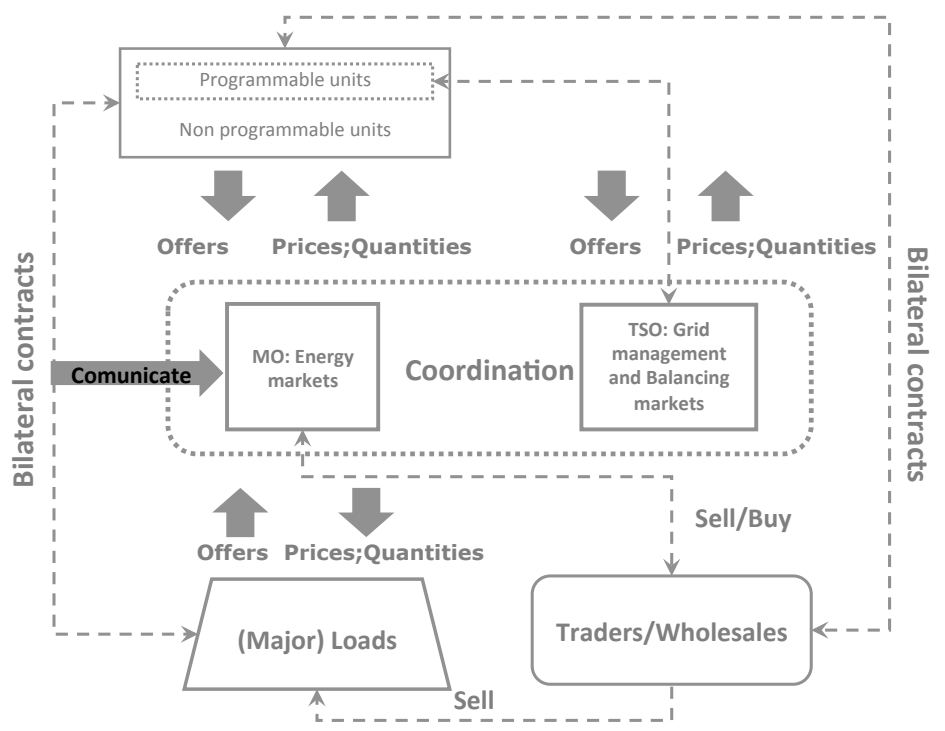

Fig. 1 Simplified electricity market structure

When the first UC models were formulated, the usual setting was that of a Monopolistic Producer (MP). The MP was in charge of the electrical production, transmission and distribution in one given area, often corresponding to a national state, comprised the regulation of exchanges with neighbouring regions. In the liberalized markets that are nowadays prevalent, the decision chain is instead decentralized and significantly more complex, as shown in the (still somewhat simplified) scheme of Figure 1. In a typical setting, companies owning generation assets (GENCOs) have to bid their generation capacity over one (or more) Market Operator(s) (MO). Alternatively, or in addition, they can stipulate bilateral contracts (or contracts for differences, CfD) with final users or with wholesales/traders. Once received the bids/offers, the MO clears the (hourly) energy market and defines (equilibrium) clearing prices. A Transmission System Operator (TSO), in possession of the transmission infrastructure, then has the duty-acting in concert with the Power Exchange Manager (PEM) - to ensure safe delivery of the energy, which in turns means different duties such as real time frequency-power balancing, spinning reserve satisfaction, voltage profile stability, and enforcing real-time network capacity constraints. The TSO typically operates 
in a different way programmable and non programmable units, since for instance only the former can participate to balancing markets.

This basic setting, which can be considered sufficient for our discussion, is only a simplification of the actual systems, which also vary depending on their geographical position. For instance, transmission (and distribution) assets may actually be in possession of different companies that have to offer them under highly regulated fair and non-discriminative conditions, leaving the TSO only a coordination role. Also, the TSO and the MO may or may not be the same entity, and so on. We leave aside these other factors, like how many and MOs there are and how exactly these are structured; we refer to \begin{tabular}{|c|c|c|}
94 & $173,281,346$ & 91.
\end{tabular} Chapter 1] for a more detailed description. Because of this complexity, standard optimization models may not be entirely appropriate to deal with all the aspects of the problem, since the behavior of different/competing decision makers need be taken into account. This may require the use of other methodologies, such as the computation of equilibria or agent-based simulation. We will not deal with any of these aspects, the interested reader being referred to $149,173,224,281,346,386$ for further discussion.

\subsection{A global view of UC}

In broad terms, the (deterministic or uncertain) Unit Commitment problem (both UC in this section unless explicitly stated) requires to minimize the cost, or maximize the benefit, obtained by the production schedule for the available generating units over a given time horizon. As such, the fundamental ingredients of UC are its objective function and its constraints. Of course, another fundamental ingredient is the time horizon itself; UC being a short-term model this is most often a day or two of operations, and up to a week. In the following we will denote it by $\mathcal{T}$, which is typically considered to be a discrete set corresponding to a finite number of time instants $t \in \mathcal{T}$, usually hours or half-hours (down to 15 or 5 minutes). Thus, the typical size of $\mathcal{T}$ varies from 24 to a few hundred.

In mathematical terms, UC has the general structure

$$
\min \left\{f(x): x \in X_{1} \cap X_{2},\right\}
$$

where $x \in \mathbb{R}^{n}$ is the decision making vector. Usually (most) elements of $x$ are indexed according to both the generating unit $i=1, \ldots, m$ and the time instant $t \in \mathcal{T}$ they refer to. Thus, one often speaks of the subvectors $x^{t}$ of all decisions pertaining to time $t$ and/or $x_{i}$ of all decisions pertaining to unit $i$. Also, entries of $x$ are typically split among:

1. commitment decision, discrete variables that determine if a particular unit is on or off at any given time (often denoted by $u_{i}^{t}$ );

2. production decision, continuous variables that provide the amount of generated power by a specific unit at a given time (often denoted by $p_{i}^{t}$ );

3. network decision, such as these representing phase angle or voltage magnitudes, describing the state of the transmission or distribution network.

A UC problem not having commitment decisions is often called Economic Dispatch (ED) (e.g. 426]) or Optimal Power Flow (OPF) when the network is considered, (e.g. 193). It could be argued that commitment decisions can be easily derived from production decisions (each time a non-zero production output is present the unit has to be on), but for modeling purposed it is useful to deal with the two different concepts separately, cf. 43.2 . Besides, the point is that in ED or OPF the commitment of units has already been fixed and cannot be changed. We remark that network decisions may also include binary variables that provide the open or close state of a particular line, as entirely closing a line is one of the few options that the physic of electrical networks allows for "routing" the electrical current (cf. $\$ 2.7$ ). While ED can be expected to be simpler than UC, and in many cases it is a simple convex program that can nowadays be solved with off-the-shelf techniques, this is not always the case. ED was not only challenging in the past (e.g., [109] and the references therein), but can still be do so today. Indeed, even 
when commitment decisions are fixed, the electrical system is highly nonlinear and nonconvex, e.g., due to hydro units efficiency curves (cf. 82.4) or the transmission network characteristics (cf. s 2.6), so that ED can still be a nontrivial problem that may require ad-hoc approaches (e.g. 185 192 193, 213, 254, 279]).

In equation (1), $X_{1}$ is the set modeling all technical/operational constraints of the individual units and $X_{2}$ are the system-wide constraints. The first set is by definition structured as a Cartesian product of smaller sets, i.e., $X_{1}=\prod_{i=1}^{m} X_{i}^{1}$, with $X_{i}^{1} \subseteq \mathbb{R}^{n_{i}}$ and $\sum_{i=1}^{m} n_{i}=n$. Moreover, the objective function $f$ typically also allows for a decomposition along the sets $X_{i}^{1}$, i.e., $f(x)=\sum_{i=1}^{m} f_{i}\left(x_{i}\right)$ and $x_{i} \in X_{i}^{1}$. Each of the sets $X_{i}^{1}$ roughly contains the feasible production schedules for one unit, that can differ very significantly between different units due to the specific aspects related to their technological and operational characteristics. In most models, $X_{1}$ is non-convex. However, units sharing the same fundamental operational principles often share a large part of their constraints as well. Because of this, these constraints are best described according to the type of the generating unit, i.e.,

1. thermal units (cf. s 2.3 ; ;

2. hydro units (cf. 22.4$) ;$

3. renewable generation units (cf. \$2.3 2.5).

While hydro units are arguably a part of renewable generation, in the context of UC it is fundamental to distinguish between those units that are programmable and those that are not. That is, hydroelectric generation systems relying on a flow that can not be programmed are to be counted among renewable generation ones together with solar and wind-powered ones. This is unless these so-called run-of-river (ROR) units are part of a hydro valley, preceded by a programmable hydro one (cf. \$2.4).

The set $X_{2}$, which usually models at least the offer-demand equilibrium constraints, is most often, but not always, convex and even polyhedral. This set may also incorporate other system-wide constraints, such as emission constraints, network transmission constraints (cf. 82.6 ) or optimal transmission switching constraints (cf. 2.7 ).

Solving (1) is difficult when $n$ is large (which usually means that $m$ is large) or $X_{1}$ is a complex set; the latter occurs e.g. when substantial modeling detail on the operations of units is integrated in the model. Finally, (1) contains no reference to uncertainty, but several sources of uncertainty are present in actual operational environments, as summarized in the following table:

\begin{tabular}{|l|l|l|}
\hline Data & Uncertain for & Severity \\
\hline customer load & GENCOs, TSO & low/medium \\
reservoirs inflows & GENCOs, TSO & medium \\
renewable generation & GENCOs, TSO & high \\
prices/quantities & GENCOs, traders, customers & medium/high \\
units/network failure & GENCOs, TSO & medium \\
\hline
\end{tabular}

Various ways to incorporate uncertainty in (1) are discussed in 4.1 . Obviously, solving (1) becomes more difficult when uncertainty is present, even when $n$ is small and $X_{1}$ relatively simple. Thus, properly exploiting the structure of the problem (the function $f$ and the sets $X_{1}$ and $X_{2}$ ) is crucial to obtain efficient schemes for UC, and even more so for UUC. This is why we now provide some detail on different modeling features for each of these components.

\subsection{The objective function}

The objective function of UC is one of the main factors reflecting the different types of decision-makers described in the previous section. In fact, when the production needs to be satisfied (as in the case of the MP, or of a GENCO having had a certain set of bids accepted) the objective function fundamentally aims at minimizing energy production costs; this is not necessarily obvious (cf. the case of hydro units below), but the principle is clear. However, in the free-market regime the aim is typically rather to 
maximize energy production profits. This again requires estimating the costs, so the same objective as in the MP case largely carries over, but it also requires estimating the revenues from energy selling, as it is the difference between the two that has to be maximized. In particular, if the GENCO is a price maker it may theoretically indulge in strategic bidding 103, whereby the GENCO withdraws power from the market (by bidding it at high cost) in order to push up market prices, resulting in an overall diminished production from its units but higher profit due to the combined effect of decreased production cost and increased unitary revenue for the produced energy. Of course, the success of such a strategy depends on the (unknown) behavior of the other participants to the market, which thereby introduces significant uncertainty in the problem. The electrical market is also highly regulated to rule out such behavior of the market participants; in particular, larger GENCOs, being more easily price makers, are strictly observed by the regulator and bid all their available capacity on the market. Yet, the solution of strategic bidding problems is of interest at least to the regulators themselves, who need to identify the GENCOs who may in principle exercise market power and identify possible patterns of abuse. Even in the price taker case, i.e., a GENCO with limited assets and little or no capacity to influence market prices, uncertainty is added by the need of accurately predicting the selling price of energy for each unit and each $t \in \mathcal{T} 156$. This uncertainty must then be managed, e.g. with techniques such as those of Robust Optimization [30].

Energy production costs for fuel-burning units are typically modeled (in increasing order of complexity) as linear, piecewise-linear convex, quadratic convex, or nonconvex functions separable for each $t \in \mathcal{T}$. In fact, while the fuel-consumption-to-generated-power curve can usually be reasonably well approximated with a piecewise linear function or a low-order polynomial one, other technical characteristics of generating systems introduce nonconvex elements. The simplest form is that of a fixed cost to be paid whenever the unit is producing at some $t \in \mathcal{T}$, irrespective of the actual amount of generated power. In alternative, or in addition, start-up costs (and, less frequently, shut-down ones) are incurred when a unit is brought online after a period of inactivity. In their simplest form start-up costs can be considered fixed, but most often they significantly depend on the time the unit has been off before having been restarted, and therefore are not separable for each time instant. The dependency of the start-up cost on time can be rather complex, as it actually depends on the choice between the unit being entirely de-powered (cooling) or being kept at an appropriate temperature, at the cost of burning some amount of fuel during the inactivity period, to make the start-up cheaper (banking). Technically speaking, in the latter case one incurs in a higher boiler cost to offset part of the turbine cost. The choice between these two alternatives can often be optimally made by simple formulæ once the amount of idle time is known, but this is typically not true beforehand in UC since the schedule of the unit is precisely the output of the optimization problem. Fortunately, some of the solution methods allow inclusion of the start-up cost at a relatively minor increase of the computational complexity; this is the case e.g. of MILP formulations, cf. §3.2, exploiting the fact that the optimal start-up cost is nondecreasing as the length of the idle period increases 75,277 ). In other cases start-up cost have basically no additional computational cost, such as in DP approaches, cf. \$3.1. Other relevant sources of nonconvexity in the objective function are valve points [406], corresponding to small regions of the feasible production levels where the actual working of the unit is unstable, e.g. due to transitioning between two different configurations in a combined-cycle unit or other technical reasons, and that therefore should be avoided.

Nuclear units are generally considered thermal plants, although they significantly differ in particular for the objective function. Indeed, fuel cost has a different structure and depends on many factors, not only technical but also political (e.g., [112]). For convenience, formulæ similar to that of conventional thermal plants are often used. However, these units incur additional significant modulation costs whenever variations of power output are required; this cost is therefore again not separable per time instant.

Hydro units are generally assumed to have zero energy production cost, although they may in principle have crew and manning costs. In the self-scheduling case, where profit has to be maximized, this would lead to units systematically depleting all the available water due to the fact that a short-term model such as UC has no "visibility" on what happens after the end of its time horizon $\mathcal{T}$ (the so-called "border effect"). Because of this, often a value of water coefficient is added to the objective function to represent the expected value of reserves left in the reservoirs at the end of $\mathcal{T}$. These values, as well as the required reservoir levels (cf.2.4), are usually computed by means of specific mid-term optimization models. A very 
standard approach is to value the differential between the initial and end volume of a reservoir against a volume-dependent water value; we refer to 80,381 for details on various other modeling choices. A particular difficulty appears when we wish to integrate the water head effect on turbining efficiency (e.g., 132,316$]$ ), since this is typically a nonlinear and nonconvex relationship.

In general, the case of profit maximization requires knowledge of the selling and buying price of energy at each $t \in \mathcal{T}$. Because UC is solved ahead of actual operations, possibly precisely with the aim of computing the bids that will contribute to the setting of these prices (cf. e.g. 60 65 210 320), this requires nontrivial forecast models in order to obtain reasonable estimates of the prices (e.g. 226 286 419]). Depending on the time horizon and specific application, different price models can be considered. These can be obtained from time series modeling (e.g. 117 264 300]), mathematical finance (e.g. [45 186 271,286 302]) or can be based on electricity fundamentals (e.g. 122,384). For the case where the producer is a price taker, that is, small enough so that its production can be deemed to have little or no effect on the realized prices, UC can typically be independently solved for each individual unit (thus being styled as the self-scheduling problem), and it is therefore much easier [16, although uncertainty in prices then becomes a critical factor 30 93 275. Things are significantly different in case the producer can exercise market power, that is, influence (increase) the prices by changing (withdrawing) the power it offers to the market; modeling this effect "ties" all the units back again into an unique UUC 65 $62,105,303$. Uncertainty in this case is also very relevant, with the behavior of competitors being one obvious primary source 7307389 396 401]. The matter is further complicated by the fact that the structure of the PE is usually complex, with more than one auction solved in cascade to account for different kinds of generation (energy, reserve, ancillary services, ...) 23 370 395 and by the fact that tight transmission constraints may create zonal or even nodal prices, thereby allowing producers who may not have market power in the global context to be able to exercise it in a limited region $[227,301,303$.

\subsection{Thermal units}

A thermal power station is a power plant in which the prime mover is steam driven. Technical/operational constraints can be classified as either static or dynamic: the former hold on each time step, whereas the latter link different (most often adjacent) time steps. Most typical static constraints are:

1. Offline: when the unit is offline, the power output is less than or equal to zero (negative power output refers to the power used by auxiliary installations, e.g., for nuclear plants).

2. Online: when the unit is online, the power output must be between Minimal Stable Generation (MSG) and maximal power output.

3. Starting: the unit is ramping up to MSG. The ramping profile depends on the number of hours a unit has been offline (e.g. [214); see also in starting curve below. A unit in this state can in principle still be disconnected for a later start, but at a cost.

4. Stopping: the unit ramps down from MSG to the offline power output. As for starting, the ramping profile depends on the number of hours a unit has been online; see below in stopping curve.

5. Generation capacity: the production capacity of each unit. For some units the production output has to be selected among a discrete set of values.

6. Spinning reserve: the extra generating capacity that is available by increasing the power output of generators that are already connected to the power system. For most generators, this increase in power output is achieved by increasing the torque applied to the turbine's rotor. Spinning reserves can be valued separately from actively generated power as they represent the main mechanism that electrical systems have to cope with real-time variations in demand levels.

7. Crew constraint: number of operators available to perform the actions in a power plant.

Typical dynamic constraints instead are:

1. Minimum Up/Down Time: a unit has to remain online/offline for at least a specific amount of time. 
2. Operating Ramp Rate (also known as ramp-down and ramp-up rate): the increment and decrement of the generation of a unit from a time step to another, excluding start-up and shut-down periods, must be bounded by a constant (possibly different for ramp-up and ramp-down).

3. Minimum Stable State Duration: a unit that has attained a specific generation level has to produce at that level for a minimum duration of time.

4. Maximum Numbers of Starts: the number of starts can be limited over a specific time horizon (such a constraint is also implicitly imposed by Minimum Up/Down Time ones, and in fact the two are often alternatives).

5. Modulation and Stability: these constraints are mainly applied to an online nuclear unit. A unit is in modulation if the output level changes in a time interval, whereas it is stable if the power level remains identical to that of the previous time step. The constraints ensure that the unit is "most often stable", requiring that the number of modulations does not exceed a predefined limit over a given time span (say, 24 hours).

6. Starting (Stopping) Curve (also referred to in literature as start-up/shut-down ramp rate): in order to start (stop) a unit and move it from the offline (online) state to the online (offline) state, the unit has to follow a specific starting (stopping) curve, which links offline power output (zero, or negative for nuclear plants) to MSG (or vice-versa) over the course of several time steps. Each starting (stopping) curve implies a specific cost, and the chosen curve depends on the number of hours the plant has been offline (online). Starting (stopping) may take anything from several minutes (and therefore be typically irrelevant) up to 24 hours (and therefore be pivotal for the schedule).

\subsection{Hydro units}

Hydro units are in fact entire hydro valleys, i.e., a set of connected reservoirs, turbines and pumps that influence each other through flow constraints. Turbines release water from uphill reservoirs to downhill ones generating energy, pumps do the opposite. Note that the power output of ROR units downstream to a reservoir (and up to the following reservoir, if any) must be counted together with that of the turbines at the same reservoir; usually it is possible to do this by manipulating the power-to-discharged-water curve of the unit at the reservoir, and thus ROR units in a hydro valley need not be explicitly modeled. We remark in passing that whether or not a unit is considered ROR depends on the time horizon of the problem: units with small reservoirs can be explicitly modeled in UC because they do have a degree of modulation over the short term, but they may be considered ROR in longer-term problems since the modulation is irrelevant over long periods of time.

As for thermal units, we distinguish constraints as being either static or dynamic. The typical ones of the first kind are:

1. Reservoir Level: the level of water in each reservoir has to remain between a lower and upper bound. Frequently these bounds are used to reflect strategic decisions corresponding to optimal long-term use of water (cf. \$2.2), and not necessarily reflect physical bounds. An alternative is to use a nonlinear cost of water that reflects the higher risk incurred in substantially depleting the reservoir level, as water in hydro reservoirs represents basically the only known way of efficiently storing energy on a large scale and therefore provides a crucial source of flexibility in the system. Yet, bounds on the level would ultimately be imposed anyway by physical constraints.

2. Bounds: turbines and pumps can operate only within certain bounds on the flowing water. In particular, some turbines might have a minimal production level akin to the MSG of thermal units.

The most common dynamic constraints are:

1. Flow Equations: these equations involve the physical balance of the water level in each reservoir and connect the various reservoirs together. The reservoir levels get updated according to natural inflows, what is turbined downhill, what is spilled downhill (i.e., let go from the reservoir to the next without activating the turbines), and what is pumped from downhill to uphill. Spilling might not be allowed for all reservoirs, nor all have pumping equipment. 
2. Flow delay: the water flowing (uphill or downhill) from each unit to the next reservoir will reach it after a given delay, that can possibly be of several hours (and occasionally even more [34]).

3. Ramp Rate: adjacent turbining levels have to remain sufficiently close to each other.

4. Smooth Turbining: over a a given time span (e.g., one hour), turbining output should not be in a $V$-shape, i.e., first increase and immediately afterwards decrease (or vice-versa). This constraint is typically imposed to avoid excessive strain on the components, similarly to several constraints on thermal units such as Minimum up/down Time, Maximum Numbers of Starts, Modulation and Stability.

5. Turbining/Pumping Incompatibility: some turbines are reversible and therefore pumping and turbining cannot be done simultaneously. Moreover, switching from turbining to pumping requires a certain delay (e.g., 30 minutes). Some of these constraints actually only refer to a single time instant and therefore they can be considered as static.

6. Forbidden Zones: in complex hydro units, effects like mechanical vibrations and cavitation strongly discourage using certain intervals of turbined water, as these would result in low efficiency and/or high output variation (similarly to valve points in thermal units, cf. \$2.2). Therefore, constraints that impose that the turbined water lies outside of these forbidden zones might have to be imposed [130].

\subsection{Renewable generation units}

Renewable generation in UC mostly refers to wind farms, solar generation, stand alone ROR hydro units, and geothermal production. The fundamental characteristic of all these sources, as far as UC is concerned, is the fact that they cannot be easily modulated: the produced energy, and even if energy is produced at all (in some wind farms energy is actually consumed to keep the blades in security when wind blows too strongly), is decided by external factors. Some of these sources, most notably solar and wind, are also characterized by their intermittency; that is, it is very difficult to provide accurate forecasts for renewable generation, even for short time horizons (say, day-ahead forecasts). Furthermore, in several cases renewable generation operates in a special regulatory regime implying that they cannot even be modulated by disconnecting them from the grid. This has (not frequently, but increasingly often) led to paradoxical situations where the spot price of energy is actually negative, i.e., one is paid to consume the energy that renewable sources have the right to produce (and sell at fixed prices) no matter what the demand actually is. All this has lead to significant changes in the operational landscape of energy production systems, that can be summarized by the following factors:

1. The total renewable production cannot be predicted accurately in advance.

2. Renewable generation has high variance.

3. The correlation between renewable generation and the load can be negative, which is particularly troublesome when load is already globally low, since significant strain is added to conventional generation assets which may have to quickly ramp down production levels, only to ramp them up (again rapidly) not much later. This goes squarely against most of the standard operational constraints in classical UC (cf. \$2.3 and $\$ 2.4$ ).

In other words, in UC terms renewable generation significantly complicates the problem; not so much because it makes its size or structure more difficult, but because it dramatically increases the level of uncertainty of net load (the load after the contribution of renewables is subtracted), forcing existing generation units to serve primarily (or at least much more often than they were designed to) as backup production in case of fluctuations, rather than as primary production systems. This increases the need of flexible (hydro-)thermal units ready to guarantee load satisfaction at a short notice, which however typically have a larger operational cost. We refer to 67,252, 261, 341, 355 for further discussion of the integration of renewable generation in UC. 


\subsection{System-wide constraints}

The most common form of system-wide constraints are the load constraints guaranteeing that global energy demand is exactly satisfied for each $t \in \mathcal{T}$. This kind of constraint is not present in the self-scheduling version of UC where each unit reacts independently to price signals, but global load satisfaction has to be taken into account, sooner or later, even in liberalized market regimes. For instance, in several countries, after the main energy market is cleared, GENCOs can swap demand between different units in order to better adjust the production schedules corresponding to the accepted bids to the operational constraints of their committed units, that are not completely represented in the auctions 318. Alternatively, or in addition, an adjustment market is ran where energy can be bought/sold to attain the same result 291,340. In both these cases the production schedules of all concerned units need be taken into account, basically leading back to global demand constraints. Also, in UC-based bidding systems the global impact of all the generation capacity of a GENCO on the energy prices need to be explicitly modeled, and this again leads to constraints linking the production levels of all units (at least, these of the given GENCO) that are very similar to standard demand constraints. Conversely, even demand constraints do not necessarily require the demand to be fully satisfied; often, slacks are added so that small amounts of deviation can be tolerated, albeit at a large cost (e.g., [119,418).

Another important issue to be mentioned is that the demand constraints need in general to take into account the shape and characteristics of the transmission network. These are typically modeled at three different levels of approximation:

- The single bus model: basically the network aspects are entirely disregarded and the demand is considered satisfied as soon as the total production is (approximately) equal to the total consumption, for each time instant, irrespectively of where these happen on the network. This corresponds to simple linear constraints and it is the most common choice in UC formulations.

- The DC model where the network structure is taken into account, including the capacity of the transmission links, but a simplified version of Kirchhoff laws is used so that the corresponding constraints are still linear, albeit more complex than in the bus model 137, 194, 218. In [15 the concept of umbrella constraints is introduced to define a subset of the network DC constraints that are active in order to significantly reduce the size of these constraints.

- The AC model where the full version of Kirchhoff laws is used, leading to highly nonlinear and nonconvex constraints, so that even the corresponding ED becomes difficult [255, 256, 265, 356, 357]. A recent interesting avenue of research concerns the fact that the non-convex AC constraints can be written as quadratic relations 192, 193, 213, which paves the way for convex relaxations using semidefinite programming approaches [254]. In particular, in the recent [187] a quadratic relaxation approach is proposed which builds upon the narrow bounds observed on decision variables (e.g. phase angle differences, voltage magnitudes) involved in power systems providing a formulation of the $\mathrm{AC}$ power flows equations that can be better incorporated into UC models with discrete variables, notably the ones of cf. 2.7 . A recount of these recent developments can be found in [55].

Although market-based electrical systems have in some sense made network constraints less apparent to energy producers, they are nonetheless still very relevant nowadays; not only in the remaining vertically integrated electrical systems, but also for the TSO that handles network security and efficiency. This requires taking into account a fully detailed network model, even considering security issues such as $N-1$ fault resilience, together with a reasonably detailed model of GENCOs' units (comprising e.g. infra-hour power ramps, start-up costs, and start-up/shut-down ramp rate), when solving the Market Balancing problem. The latter is basically a residual demand, bidding-based UC. From a different perspective, network constraints might also be important for GENCOs that are able exercise market power in case zonal or nodal pricing is induced by the network structure 312 .

Finally, both for vertically integrated system and in the TSO perspective, other relevant system-wide constraints are spinning reserve ones: the committed units must be able to provide some fraction (at least $3 \%$ according to [367]) of the total load in order to cope with unexpected surge of demand or failures of generating units and/or transmission equipment. Other global constraints linking all units, 
or some subsets of them, exist: for instance, all (or specific subsets of) fossil-fuel burning units may have a maximum cap on the generation of pollutants $\left(\mathrm{CO}_{2}, \mathrm{SO}_{x}, \mathrm{NO}_{x}\right.$, particles, ...) within the time horizon 148, 158, 190,209,399. Alternatively, a cluster of geographically near units (a plant) burning the same fuel (typically gas) may be served by a unique reservoir, and can therefore share a constraint regarding the maximum amount of fuel that can be withdrawn from the reservoir within the time horizon 11, 12, 87, 148, 369. Finally, there may be constraints on the minimum time between two consecutive start-ups in the same plant 119, e.g., due to crew constraints. If a plant comprises a small enough number of units it could alternatively be considered as a single "large" unit, so that these constraints become technical ones of this aggregated generator. The downside is that the problem corresponding to such a meta-unit then becomes considerably more difficult to solve.

\subsection{Optimal Transmission Switching}

Traditionally, in UC models the transmission network has been regarded as a "passive" element, whose role was just to allow energy to flow from generating units to demand points. This is also justified by the fact that electrical networks, unlike most other networks (logistic, telecommunications, gas, water, ... ) are "not routable": the current can only be influenced by changing nodal power injection, which is however partly fixed (at least as demand is concerned). Indeed, in traditional UC models there were no "network variables", and the behavior of the transmission system was only modeled by constraints. However, as the previous paragraph has recalled, the transmission network is by far not a trivial element in the system, and separate network variables are required. Recently, the concept has been further extended to the case where the system behavior can be optimized by dynamically changing the topology of the network. This is a somewhat counterintuitive consequence of Kirchhoff laws: opening (interrupting) a line, maybe even a congested one, causes a global re-routing of electrical energy and may reduce the overall cost, e.g. by allowing to increase the power output of some cheaper (say, renewable) units [134]. This effect can be especially relevant in those parts of the network with a high fraction of renewables whose production is sometimes cut off because of network constraints.

Thus, a new class of problems, called Optimal Transmission Switching (OTS) or System Topology Optimization (STO), has been defined whereby each line of the network has an associated binary decision (for each $t \in \mathcal{T}$ ) corresponding to the possibility of opening it. This makes the problem difficult to solve even with a very simple model of nodal injections and a simple network model such as the DC one (cf. \$2.6); even more so with the AC model and a complete description of the generating units. The so-called UCOTS models $56,134,174,177,207,232,233,243,280,284,285,298,327,388,420$ extend UC: almost everything that can be said about UC is a fortiori valid for UCOTS, and therefore in the following we will not distinguish between the two unless strictly necessary.

\section{Methods for the deterministic Unit Commitment}

We now proceed with a survey of solution methods for (the deterministic) UC. Our choice to first focus on the case where the several forms of uncertainty arising in UC (cf. 82.1 are neglected is justified by the following facts:

- UC already being a rather difficult problem in practice, most work has been carried out in the deterministic setting;

- uncertainty can be taken into account through various "engineering rules": for instance, spinning reserves allow to account for uncertainty on load, tweaking reservoir volumes might allow to account for uncertainty on inflows, and so on;

- methods for solving the deterministic UC are bound to provide essential knowledge when dealing with UUC. 
As discussed in Section 2, UC is not one specific problem but rather a large family of problems exhibiting common features. Since the set of constraints dealt with in the UC literature varies from one source to another, we define what we will call a basic Unit Commitment problem (bUC) which roughly covers the most common problem type; through the use of tables we will then highlight which sources consider additional constraints. A bUC is a model containing the following constraints:

1. offer-demand equilibrium;

2. minimum up or down time;

3. spinning reserve;

4. generation capacities.

The UC literature review 349, of which 290 is essentially an update adding heuristic approaches, generally classify UC methodology in roughly eight classes. We will essentially keep this distinction, but regroup all heuristic approaches in "Meta-Heuristics", thus leading us to a classification in:

1. Dynamic Programming;

2. MILP approaches;

3. Decomposition approaches;

4. (Meta-)Heuristics approaches.

We will also add some of the early UC approaches in the Heuristic class such as priority listing. However, we will not delve much on that class of approaches, since the recent surveys [127, 337] mainly focus on these, while providing little (or no) details on approaches based on mathematical programming techniques, that are instead crucial for us in view of the extension to the UUC case.

\subsection{Dynamic Programming}

Dynamic Programming (DP, see e.g. 33, 49,50$]$ ) is one of the classical approaches for UC. As discussed below, it is nowadays mostly used for solving subproblems of UC, often in relation with Lagrangianbased decomposition methods (cf. \$3.3); however, attempts have been made to solve the problem as a whole. There have been several suggestions to overcome the curse of dimensionality that DP is known to suffer from; we can name combinations of DP and Priority Listing (DP-PL) [189, 361], Sequential Combination (DP-SC) [293, Truncated Combination (DP-TC) 292], Sequential/Truncated Combination (DP-STC) (the integration of the two aforesaid methods) 293], variable window truncated DP 287], approximated DP [104] or even some heuristics such as the use of neural network [287] or artificial intelligence techniques 392. The multi-pass DP approach 124 416] consists of applying DP iteratively, wherein in each iteration the discretization of the state space, time space and controls are refined around the previously obtained coarse solution; usually, this is applied to ED, i.e., once commitment decisions have been fixed. In [293 three of the aforesaid methods, DP-PL, DP-SC, and DP-STC are compared against a priority list method on a system with 96 thermal units, showing that the DP-related approaches are preferable to the latter in terms of time and performance. The recent 359 performs a similar study on a bUC with 10 thermal units, but only DP approaches are investigated.

Despite its limited success as a technique for solving UC, DP is important because of its role in dealing with sub-problems in decomposition schemes like Lagrangian relaxation. These typically relax the constraints linking different unit together, so that one is left with single-Unit Commitment (1UC) problems, i.e., self-scheduling ones where the unit only reacts to price signals. In the "basic" case of time-independent startup costs $1 \mathrm{UC}$ can be solved in linear time on the size of $\mathcal{T}$. When dealing with time-dependent startup costs instead, this cost becomes quadratic 29, 427. However, this requires that the optimal production decisions $p_{t}^{i}$ can be independently set for each time instant if the corresponding commitment decision $u_{t}^{i}$ is fixed, which is true in bUC but not if ramp rate constraints are present. It is possible to discretize power variables and keep using DP 32 , but the approach is far less efficient and the determined solution is not guaranteed to be feasible. An efficient DP approach for the case of ramp rate 
constraints and time-dependent startup costs has been developed in [126] under the assumption that the power production cost is piecewise linear. This has been later extended in 142 for general convex cost functions; under mild conditions (satisfied e.g., in the standard quadratic case), this procedure has cubic cost in the size of $\mathcal{T}$. DP has also been used to address hydro valley subproblems in 360 where a three stage procedure is used: first an expert system is used to select desirable solutions, then a DP approach is used on a plant by plant basis, and a final network optimization step resolves the links between the reservoirs. In 334 expert systems and DP are also coupled in order to solve UC. We also mention the uses of expert systems in 253 .

Most often DP approaches are applied to bUC, but other constraints have been considered such as multi-area, fuel constraint, ramp rates, emission constraints, and hydro-thermal systems. We refer to Table 1 for a complete list.

Table 1 Sources using Dynamic Programming

\begin{tabular}{|c|c|c|c|c|c|c|c|c|c|}
\hline \multirow[t]{2}{*}{ Basic UC } & \multicolumn{9}{|c|}{ Additional UC constraints } \\
\hline & $\begin{array}{l}\text { Must } \\
\text { Run/Off }\end{array}$ & $\begin{array}{l}\text { Fixed } \\
\text { Generation }\end{array}$ & $\begin{array}{l}\text { Crew } \\
\text { Constr. }\end{array}$ & $\begin{array}{l}\text { Ramp } \\
\text { Rate }\end{array}$ & $\begin{array}{l}\text { Operating } \\
\text { Reserve }\end{array}$ & $\begin{array}{l}\text { Maint- } \\
\text { nance }\end{array}$ & $\begin{array}{l}\text { Hydro } \\
\text {-Thermal }\end{array}$ & $\begin{array}{l}\text { Fuel } \\
\text { Const. }\end{array}$ & Emission \\
\hline 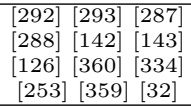 & 292 & 292 & 292 & $\frac{142}{\frac{1426}{392}}$ & 360 & 253 & $143 \quad 360$ & 4 & 190 \\
\hline
\end{tabular}

\subsection{Integer and Mixed Integer Linear Programming}

\subsubsection{Early use: exhaustive enumeration}

As its name implies, this approach focuses on a complete enumeration of the solution space in order to select the solution with the least cost. bUC is addressed in [172, 204], while in 172 the cost function considers penalties for loss of load and over production. In 204] a set of 12 thermal units on a two hour basis is scheduled. In 172 a problem with two groups, each of which has 5 thermal units is analyzed. This traditional approach obviously lacks scalability to large-scale systems. However, some enumeration may find its way into hybrid approaches such as decomposition methods under specific circumstances, like in 132 where enumeration is used in some of the subproblems in a decomposed hydro valley system.

\subsubsection{Modern use of MILP techniques}

With the rise of very efficient MILP solvers, MILP formulations of UC have become common. In general, their efficiency heavily depends on the amount of modeling detail that is integrated in the problem. Early applications of MILP can be found in [88, 151, 263], and in [88 it is stated that the model could be extended to allow for probabilistic reserve constraints. Hydro-thermal UC is considered in 114 304 348 where constraints regarding hydro units such as flow equations, storage level of reservoirs, pump storage and min and max of outflow of each reservoir are incorporated in the model.

Some specific constraints such as the number of starts in a day or particular cost functions with integrated banking costs can be found in 212,376 . In 212 the authors combine Lagrangian relaxation (e.g., 262 ) with a $\mathrm{B} \& \mathrm{~B}$ procedure in order to derive valid bounds to improve the branching procedure. The upper bound is derived by setting up a dynamic priority list in order to derive feasible solutions of the UC and hence provide upper bounds. It is reported that a 250 unit UC was solved up to $1 \%$ of optimality in less than half an hour, a significant feat for the time. A similar approach is investigated in 299, where a heuristic approach using, among things, temporal aggregation is used to produce a good quality integer feasible solution to warm-start a B\&B procedure.

While MILP is a powerful modeling tool, its main drawback is that it may scale poorly when the number of units increases or when additional modeling detail is integrated. To overcome this problem it 
has been combined with methods such as DP [61, logic programming [191] and Quadratic Programming (QP) 345]. In 345 a hydro-thermal UC with various constraints is solved; a customized B\&B procedure is developed wherein binary variables are branched upon according to their difference from bounds. The approach does not require any decomposition method, and it is reported to reduce solution time significantly in comparison to other methods. The paper builds upon [147, where a six-step solution is proposed to solve large-scale UC; the algorithm is reported to be capable of solving security-constrained problems with 169, 676 and 2709 thermal units in 27s, 82s and 8 minutes, respectively. This so-called Fast-Security Constraint Unit Commitment problem (F-SCUC) method is based on an ad-hoc way of fixing binary variables and gradually unlock them if needed, using Benders-type cuts to this effect. However, in 143 it is reported that MILP models where the objective function is piecewise-linearly approximated are much more effective than the direct use of MIQP models, at least for one specific choice and version of the general-purpose MIQP solver. In 145 MILP and Lagrangian methods are combined, solving problems with up to 200 thermal units and 100 hydro units in a few minutes if the desired accuracy is set appropriately.

Systems with a significant fraction of hydro generation require a specific mention due to a notable characteristic: the relationship between the power that can be generated and the level of the downstream reservoir (head-to-generated-power function), that can be highly nonlinear [76], and in particular nonconvex. This can be tackled by either trying to find convex formulations for significant special cases 417], developing ad-hoc approximations that make the problem easier to solve 77, or using the modeling features of MILP to represent this (and other nonconvex) feature(s) of the generating units 83.306. However, developing a good approximation of the true behavior of the function is rather complex because it depends on both the head value of the reservoir and the water flow. MILP models for accurately representing this dependency have been presented in [197, and more advanced ones in [63 using ideas from [98]; while they are shown to significantly improve the quality of the generated schedules, this feature makes UC markedly more complex to solve.

\subsubsection{Recent trends in MILP techniques}

Recently, MIP (and in particular MILP) models have attracted a renewed attention due to a number of factors. Perhaps the most relevant is the fact that MILP solvers have significantly increased their performances, so that more and more UC formulations can be solved by MILP models with reasonable accuracy in running times compatible with actual operational use 75 . Furthermore, selected nonlinear features - in particular convex quadratic objective functions and their generalization, i.e., Second-Order Cone Constraints - are nowadays efficiently integrated in many solvers, allowing to better represent some of the features of the physical system. This is especially interesting because MIP models are much easier to modify than custom-made solution algorithms, which - in principle - allow to quickly adapt the model to the changing needs of the decision-makers. However, it has to be remarked that each modification to the model incurs a serious risk of making the problems much more difficult to solve. Two somewhat opposite trends have recently shown up. On one side, tighter formulations are developed that allow to more efficiently solve a given UC problem because the continuous relaxation of the model provides better lower bounds. On the other hand, more accurate models are developed which better reflect the real-world behavior of the generating units and all the operational flexibility they possess (cf. e.g. [188, 236, 245]), thereby helping to produce better operational decisions in practice.

On the first stream, the research has focused on finding better representations of significant fragments of UC formulations. For instance, 257,282 develop better representations of the polyhedra describing minimum up- and down-time constraints and ramping constraints, whereas 144 196 408 focus on better piecewise-linear reformulations of the nonlinear (quadratic) power cost function of thermal units. Both approaches (that can be easily combined) have been shown to increase the efficiency of the MILP solver for a fixed level of modeling detail.

The second stream rather aims at improving the accuracy of the models in representing the real-world operating constraints of units, that are often rather crudely approximated in standard UC formulations. For hydro units this for instance concerns technical constraints 83 and the already discussed water- 
to-produced-energy function, with its dependency from the water head of the downstream reservoir 63, 132, 306]. For thermal units, improvements in the model comprise the correct evaluation of the power contribution of the start-up and shut-down power trajectories (when a unit is producing but no modulation is possible) [17, which may make the model significantly more difficult unless appropriate techniques are used [258], or a clearer distinction between the produced energy and the power trajectory of the units $[150,259]$.

In the OTS context (cf. $\S 2.7$ ), special care must be given when modeling the Kirchhoff laws, as this leads to logic constraints that, in MILP models, are typically transformed into "Big-M" (hence, weak) linear constraints. Moreover, severe symmetry issues 283 must be faced 243.285, as these can significantly degrade the performances of the B\&B approach. All these difficulties, not shared by UC with DC or AC network constraints, require a nontrivial extension of the "classic" MILP UC models. Many approaches use off-the-shelf B\&B solvers, while possibly reducing the search space of the OTS binary variables [233, 284, 327] and using tight formulations for the thermal units constraints. All the references use classic quadratic cost functions; one exception can be found in 243], where a direct MILP approach is combined with a perspective cuts approximation 144 and a special perturbation of the cost function that successfully breaks (part of the) symmetries. Together with heuristic branching priorities that give precedence to the thermal UC status variables, this is shown to be much better than using a classic quadratic function, with or without perturbations, for solving the IEEE 118 test case.

Table 2 Sources using MILP approaches

\begin{tabular}{|c|c|c|c|c|c|c|c|c|c|c|c|}
\hline \multirow[t]{2}{*}{ Basic UC } & \multicolumn{11}{|c|}{ Additional UC constraints } \\
\hline & $\begin{array}{l}\text { Must } \\
\text { Run/Off }\end{array}$ & $\begin{array}{l}\text { Trans } \\
\text {-OTS }\end{array}$ & $\begin{array}{l}\text { Modul- } \\
\text {-ation }\end{array}$ & Starts & $\begin{array}{l}\text { Hot/Cold } \\
\text { Starts }\end{array}$ & $\begin{array}{l}\text { Ramp } \\
\text { Rate }\end{array}$ & $\begin{array}{l}\text { Hydro- } \\
\text { Thermal }\end{array}$ & $\begin{array}{l}\text { Water- } \\
\text { head }\end{array}$ & $\begin{array}{l}\text { Thermal- } \\
\text { Stress }\end{array}$ & Fuel & Emission \\
\hline 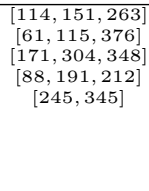 & $\frac{\overline{114}}{\frac{144}{145}}$ & 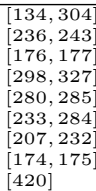 & 114 & 376 & 212 & \begin{tabular}{|l|l|}
191 & 345 \\
75 & 145 \\
230 & 282 \\
144 & 257 \\
17 & 196 \\
150 & 258 \\
150 & 259 \\
\end{tabular} & \begin{tabular}{|l|l|}
$\begin{array}{l}345 \\
114\end{array}$ & 348 \\
145 & $\frac{304}{214}$ \\
1444 & \\
\end{tabular} & 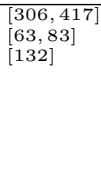 & 228 . & 236 & 236 \\
\hline
\end{tabular}

\subsection{Lagrangian and Benders Decomposition}

UC possesses several forms of structure that can be algorithmically exploited; the most obvious one is that (complex) units are usually coupled through demand and reserve requirements (the set $X_{2}$ in (1)). Since these constraints are usually in limited number and "simple", Lagrangian Decomposition (or Relaxation, LR) $140,167,220$ is an attractive approach and has been widely used. It is based on relaxing these coupling constraints by moving them in the objective function, weighted by appropriate Lagrangian multipliers, so that the relaxed problem then naturally decomposes into independent subproblems for each individual unit (1UC); for an arbitrary set of Lagrangian multipliers, the solution of all the 1UCs provides a lower bound on the optimal value of (1). Moreover the mapping (called the dual function, or Lagrangian function) assigning this optimal value to a given set of Lagrangian multipliers is concave; maximizing it, i.e., finding the best possible lower bound, is therefore a convex optimization problem for which efficient algorithms exists.

Two technical points are crucial when developing a LR approach:

- how the maximization of the Lagrangian function, i.e., the solution of the Lagrangian Dual (LD), is performed;

- since (1) is in general nonconvex the approach cannot be expected to provide an optimal (or even feasible) solution, so methods to recover one have to be developed.

Regarding the first point, one can rely on the available well-developed theory concerning minimization of convex nondifferentiable functions. Standard approaches of this kind are subgradient meth- 
ods 100,270,308 and the cutting plane method (CP) 203, also known as the Dantzig-Wolfe decomposition method 101]. Early examples of the use of subgradient methods in UC are [29, 47, 135 248, 262 427], possibly with modifications such as successive approximation techniques 87 or variable metric approaches 12. An early example of the use of $\mathrm{CP}$ is $[2$. The two approaches are rather different: subgradient methods use very simple rules to compute the next dual iterate, whereas CP uses (possibly costly) Linear Programming (LP) problems for the same task, although hybrid versions have been devised 369. This is necessary in practice because both approaches have convergence issues, for different reasons: subgradient methods lack an effective stopping criterion, whereas CP tends to be unstable and converge slowly. This is why variants of CP have been devised, e.g., using Interior Point ideas to provide some stabilizing effect 118; for an application to UC see [244]. In 332 the KKT conditions of the Lagrange function are used in order to update the Lagrange multipliers and improve on subgradient approaches. In 319 $\mathrm{CP}$ is stabilized by a trust region. The latter turns out to be a special case of the most effective family of approaches capable of dealing with this kind of problems, that is, (generalized [139]) Bundle methods 219 402. These can be seen as a "mix" between subgradient and CP 22 which inherits the best properties of both [68. Several variants of Bundle approaches exist, see e.g. [18, 221,222]; a recent development that is particularly useful for UC is that of methods that allow the inexact solution of the Lagrangian relaxation 106, 107,206. This feature is of particular interest if operational considerations impose strong restrictions on the solution times for the subproblems. For early application of Bundle methods to UC see e.g., 64, 65, 128, 159,223, 242, 421].

Regarding the second point, one important property of LDs of non-convex programs is that, while they cannot be guaranteed to solve the original problem, they indeed solve a "convexified version" of it 140 220. In practice, this typically corresponds to a solution $\tilde{x}=(\tilde{p}, \tilde{u})$ to (1) that is feasible for all constraints except the integrality ones. That is, rather than feasible commitment decisions $u_{t}^{i} \in\{0,1\}$ one obtains pseudo-schedules $\tilde{u}_{t}^{i} \in[0,1]$ that satisfy the constraints with the production decisions $\tilde{p}$. Such a solution can be obtained basically for free by (appropriately instrumented versions of) subgradient methods $[10,28$ and all other algorithms, most notably Bundle ones [128. The pseudo-schedule $\tilde{x}$ can for instance be heuristically interpreted as the probability that unit $i$ be on at instant $t$, and then be used in this guise to devise primal recovery approaches to attain feasible solutions of (1), either by appropriately modifying the objective function 99,119 or by a heuristic search phase that exploits both $\tilde{x}$ and the integer solutions produced by the LR [31, 143] 333 .

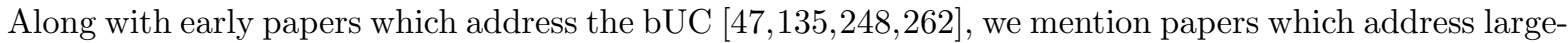
scale UC [4, 248]. The authors of 248 are among the first who tried to use LR to obtain a solution, and not just to obtain lower bounds for B\&B procedures, solving a problem of 172 units. In 212 the duality gap problem is tackled by approximating the dual problem with a twice-differentiable mapping which is then maximized by using a constrained Newton's method, after which a heuristic is used to recover a nearly optimal primal solution; a 200 units UC is solved in about 10 to 12 minutes. In a subsequent work [348], a three-stage approach is proposed to deal with a-for the time-large-scale hydro-thermal system (100 thermal units and 6 hydro ones). The first stage is based on LR, with the thermal 1UCs solved using DP, while the hydro subproblems are solved by using a penalty multipliers method 208 and a specially tailored Newton's method. A "unit decommitment" method is suggested in 225,373 where all units are considered online over all $\mathcal{T}$ and then, using the results of the LR, units are decommitted one at a time. This method aims at providing feasible primal solutions first, whereas most LR approaches would aim at optimality first. Further references using LR are 129, 164, 335, 336], which consider specific dedicated approaches in order to tackle the subproblems, elementary ways of updating the dual and heuristics to recover a primal feasible solution. In 162 the units cost functions are modified in order to reduce the oscillating behavior of subgradient approaches. In $[159$ the authors compare a primal MIP based approach with a LR-based approach: Bundle methods are used in order to solve the LD and two Lagrangian heuristics are investigated for primal recovery. The first one searches for time steps where demand constraints are most violated and employs a strategy proposed in 427 for changing the commitment variables, while the second one exploits nearly optimal Lagrange multipliers for fixing commitment decisions. In order to recover primal feasibility, both heuristics are followed by solving an ED, wherein the commitment variables are fixed; this LR-based method is shown to be capable of handling larger and more complex instances. In [366] the Lagrangian heuristic consists of formulating 
a MIP that mixes solutions provided by the dual iterations, selecting the production schedule of a specific unit among the primal solutions generated by the LD phase in such a way as to minimize

Table 3 Sources using Lagrangian Relaxation

\begin{tabular}{|c|c|c|c|c|c|c|c|}
\hline \multirow[t]{2}{*}{ Basic UC } & \multicolumn{7}{|c|}{ Additional UC constraints } \\
\hline & $\begin{array}{l}\text { Must } \\
\text { Run/Off }\end{array}$ & $\begin{array}{l}\text { Fuel } \\
\text { Constr. }\end{array}$ & $\begin{array}{l}\text { Ramp } \\
\text { Rate }\end{array}$ & $\begin{array}{l}\text { Suppl. } \\
\text { Reserve }\end{array}$ & $\begin{array}{l}\text { Hydro- } \\
\text { - Thermal }\end{array}$ & Emission & Transmission \\
\hline 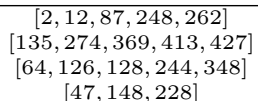 & \begin{tabular}{|l|l|}
$\overline{413}$ & 427 \\
145 & \\
\end{tabular} & $\overline{12} \frac{\overline{369}}{148}$ & \begin{tabular}{|l|l|}
87 & 148 \\
143 & $\overline{143}$ \\
65 & 66 \\
11 & 228 \\
\end{tabular} & $\overline{2} \mid \overline{87}$. & \begin{tabular}{|l|l|l|}
12 & $\overline{365}$ & $\frac{413}{145}$ \\
66 & $\frac{143}{145}$ \\
$\overline{65}$ & $\frac{274}{348}$ \\
11 & $\frac{341}{131}$ & $\overline{132}$ \\
\end{tabular} & $\overline{148} \overline{158} \overline{209}$ & \begin{tabular}{|l|l|}
2 & 364 \\
\end{tabular} \\
\hline
\end{tabular}


A different decomposition approach is the classic one due to Benders 44 [62, Chapter 11.1], which rather focuses on complicating variables that, once fixed, allow to separate the problem into independent (and, hopefully, easy) ones. Application of Benders' decomposition to UC is fairly recent. In 231, 407 techniques for improving the Benders' cuts production are described. In 146 a conceptual and numerical comparison is made, in the context of the security constrained UC, between LR and MILP

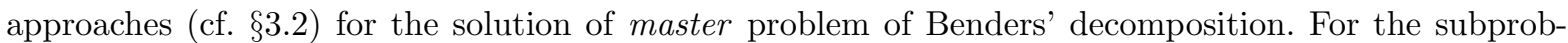
lems, involving the network constraints, the authors compare Benders' cuts and linear sensitivity factor (LSF) approaches.

\subsection{Augmented Lagrangian Relaxation}

One major downside of LR approaches is the difficulty in recovering a primal feasible solution. The use of the Augmented Lagrangian (AL) method, whereby a quadratic penalization of the relaxed constraints is added to the objective function alongside the linear penalization typical of standard LR, is known to be a potential solution to this issue. Yet, because (1) is nonconvex it should be expected that in general the AL approach leads to a local optimizer [157,240]. Furthermore, the AL relaxation is no longer separable into an independent subproblem for each unit, and therefore it is significantly more difficult to solve (in practice, as difficult as UC itself). This calls for some further approach to simplify the relaxation; in 31, 414 the use of the auxiliary problem principle 89,90 is suggested. The classic theory of the auxiliary problem principe requires restrictive assumptions such as convexity and regularity, which do not hold in practice; some recent advances have been made in the non-convex setting $19,317,374$. In 35 an alternative decomposition scheme based on block coordinate descent (e.g. [48, 328]) is proposed and it is found to be more efficient. The recent [249] includes in the UC formulation a DC network model and bilateral contracts defining the nodal injections. The AL of the coupling constraints is formed and then linearized in an ad-hoc way, while Bundle methods are employed for updating the dual multipliers. Environmental constraints [399] and network transmission constraints [35, 399 have also been tackled with the AL approach. A common way to deal with additional constraints is variable duplication [153.

Table 4 Sources using Augmented Lagrangian Approaches

\begin{tabular}{|c|c|c|c|c|c|c|}
\hline \multirow[t]{2}{*}{ Basic UC } & \multicolumn{6}{|c|}{ Additional UC constraints } \\
\hline & Modulation & $\begin{array}{l}\text { Startup/shutdown } \\
\text { curves }\end{array}$ & Transmission & $\begin{array}{l}\text { Ramp } \\
\text { Rate }\end{array}$ & $\begin{array}{l}\text { Environ. } \\
\text { Const. }\end{array}$ & $\begin{array}{l}\text { Hydro- } \\
\text {-Thermal }\end{array}$ \\
\hline $25 \quad 3135 \quad 399$ & 31 & 31 & $\begin{array}{lll}25 & 35 & 399\end{array}$ & 2531 & 399 & 25 \\
\hline
\end{tabular}

\section{5 (Meta-)Heuristics}

\subsubsection{Operator rule based: Priority Listing}

This method defines a list of units which should logically be scheduled prior to other units, with merit order scheduling being a special case. Priority listing was first employed on bUC in [26, where units are listed according to their performance and the cost they yield (comprising maintenance costs). Muston/must-off and crew constraint have been added in 215, and a limit on the number of starts is included in 216 through the use of a commitment utilization factor, which is claimed to provide a better list. While the former two papers and [5] address bUC, there has been an endeavour to integrate other factors such as multi-area constraints [217] and hydro-thermal systems [200] for large-scale UC. In the latter paper a two-step heuristic procedure is used to solve a UC with 100 units: the first step uses rules from real-world schedules (possibly enhanced by the use of UC software) to set up a priority list consisting of feasible production schedules, while the second step optimizes locally around the current solution. A very similar approach is investigated in [5]. 
Table 5 Sources using Priority Listing

\begin{tabular}{|c|c|c|c|c|c|c|}
\hline \multirow[t]{2}{*}{ Basic UC } & \multicolumn{6}{|c|}{ Additional UC constraints } \\
\hline & $\begin{array}{l}\text { No.Units } \\
\text { Started }\end{array}$ & $\begin{array}{l}\text { Crew } \\
\text { Const. }\end{array}$ & $\begin{array}{l}\text { Must } \\
\text { run/Off }\end{array}$ & $\begin{array}{l}\text { Multi- } \\
\text { Area }\end{array}$ & $\begin{array}{l}\text { Hydro- } \\
\text { Thermal }\end{array}$ & $\begin{array}{l}\text { No. Starts } \\
\text { / Shutdowns }\end{array}$ \\
\hline \begin{tabular}{l|l|l}
5 & $\overline{26}$ & $\overline{200}$
\end{tabular} & 200 & 215 & $\overline{215} \mid \overline{217}$ & 217 & 200 & 216 \\
\hline
\end{tabular}

\subsubsection{Guided Random Exploration}

Since solving the UC (1) to optimality is quite difficult, many heuristic approaches such as Taboo search, Simulated Annealing, Augmented Lagrange Hopfield Networks, Nature Inspired (e.g., particle swarms, frog leaping, ...) and Genetic Algorithms have also been employed. We refer to 127,337 for a discussion of those approaches, and in this paper we by no means attempt to give a full overview of this subfield. This is because heuristic approaches like these are typically difficult to adapt to the Uncertain UC case, which is the main focus of this survey, unless they are at least partly based on mathematical programming techniques. We therefore concentrate mostly on "hybrid" approaches that use the latter at least to a certain degree. For instance, in 237] genes are feasible schedules produced by a LR-based scheme: the genetic algorithm then mixes the solutions up to form new feasible schedules in order to hopefully produce a solution that better meets the demand constraints. In 428 the authors solve a 100 thermal unit system by using Simulated Annealing and report that their approach outperforms a B\&B procedure, but fails to outperform a LR approach (although in the later 64 Taboo search has been reported to be more competitive with LR). In [120 201] Evolutionary Programming is applied to adjust the solution provided by a LR approach. In 241] a neural network approach is coupled to LR in order to optimize a system with up to 60 units: the thermal subproblems are optimized using a neuron-based DP algorithm.

In general, these approaches are not considered particularly competitive for UC; for instance, 368 states that Simulated Annealing and Evolutionary Programming attempts have been unsuccessful. Also, usually these approaches deal with bUC, with only a few sources considering ramp rate, crew, maintenance or multi-area constraints, and hydro-thermal systems being very rarely dealt with. The likely reason is that purely combinatorial heuristics are best apt at problems that exhibit a predominant and relatively "simple" combinatorial structure to which the various elements of the heuristic (neighborhood(s) structure in Simulated Annealing, Taboo list and aspiration criteria in Taboo search, mutation and crossover operators in genetic algorithms, ...) can be specifically tailored. UC is a fundamentally mixed combinatorial and continuous program, since both the commitment and the dispatch have to be provided. Furthermore, UC has several different combinatorial structures, especially when "complex" constraints have to be dealt with. Therefore, on the outset UC is best approached with mathematical programming techniques.

Table 6 provides a (very partial) overview of heuristic approaches:

Table 6 Sources using (Meta-)Heuristic Approaches

\begin{tabular}{|c|c|c|c|c|c|c|c|}
\hline \multirow[t]{2}{*}{ Approach } & \multirow[t]{2}{*}{ Basic UC } & \multicolumn{6}{|c|}{ Additional UC constraints } \\
\hline & & $\begin{array}{l}\text { Ramp } \\
\text { Rate }\end{array}$ & $\begin{array}{l}\text { Crew } \\
\text { Constr. }\end{array}$ & $\begin{array}{l}\text { Mainte- } \\
\text { nance }\end{array}$ & $\begin{array}{l}\text { Multi-Area } \\
\text { Const. }\end{array}$ & $\begin{array}{l}\text { Hydro- } \\
\text { Thermal }\end{array}$ & Derating \\
\hline Simul. Annealing & $\frac{8358,428}{246}$ & 358 & \begin{tabular}{|l|l|l|l|l|l|l|} 
& 246 & 428 \\
\end{tabular} & 428 & & & \\
\hline Tabu Search & 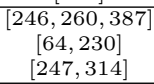 & & 246 & & & 247 & 246 \\
\hline Neural Network & \begin{tabular}{|l|l|l|}
339 & & \multicolumn{1}{|c|}{} \\
1 & 343 & 391 \\
113 & 324 & 392 \\
113 & 266 \\
\end{tabular} & \begin{tabular}{|l|l|l|}
1 & 343 \\
113 & 392 \\
\end{tabular} & 266 & & & 391 & \\
\hline Genetic Algorithm & \begin{tabular}{|l|l|l|}
363 & 403 & 404 \\
86 & 378 & 415 \\
313 & 315 & 350 \\
120 & 201 & 415 \\
& 102 & 237 \\
\end{tabular} & \begin{tabular}{|l|l|}
350 & 403 \\
313 & \\
\end{tabular} & & & \begin{tabular}{|l|l|}
86 & 315 \\
\end{tabular} & & \\
\hline Nature Inspired & $81 \quad \overline{82} \overline{152}$ & $\begin{array}{lll}81 & 82 & 152 \\
\end{array}$ & & & 82 & & \\
\hline
\end{tabular}




\section{Methods for the Uncertain Unit Commitment}

The complex nature of UC, due to its numerous technical constraints, forces the schedule to be determined quite ahead of time and consequently be given to the TSO one day in advance. This allows for uncertainty to have an important impact on the system. Furthermore, intra-daily optimization processes and communication between the TSO and the GENCOs allow for recourse decisions. Thus, dealing with uncertainty has always been necessary in UC. We now discuss the approaches that have been proposed in the literature. To the best of our knowledge, this has never been done before specifically for the UC. The chapter 390 provides a general overview of the ways in which uncertainty arises in Energy Management, but it is mainly focused on mid- and long-term problems, UC being only briefly addressed. Analogously, 91 offers a general survey on uncertainty issues in Energy Optimization, without a specific focus on UC. The chapter 325] offers a general overview of properties of stochastic optimization problems and briefly provides some links to stochastic UC problems. The essential references used in these sources will be discussed below.

\subsection{Dealing with Uncertainty in UC}

In most traditional approaches, load uncertainty is dealt with by computing the schedule corresponding to the worst scenario, i.e., typically that of peak demand in each period. This choice systematically overestimates demand and incurs the risk that significant ramp-down of the production is needed when the actual demand proves to be substantially smaller than the forecasted one, which can cause feasibility issues due to technical constraints like ramp-down ones (cf. 2.3 . Another common approach has been to

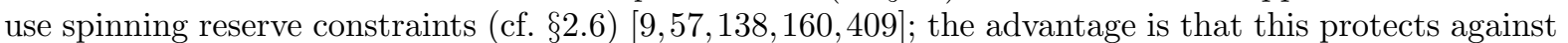
some degree of uncertainty while keeping the deterministic formulation. In general, the deterministic constraints can be "tweaked" heuristically in order to deal with uncertainty. For instance, in order to ensure that the solution can survive a certain degree of variability in the data we can underestimate the amount of water in a hydro reservoir and/or impose stricter ramp-rate constraints than justified by technical aspects. Obviously, this may result in a loss of optimality or control over feasibility. Worse, one may loose control over where the approximations have been made.

In order to overcome these weaknesses, methods where uncertainty is directly modeled have been investigated. These comprise Stochastic Optimization (scenario tree), Robust Optimization, and ChanceConstrained Optimization.

\subsubsection{Dealing with uncertainty in the model}

4.1.1.1 Stochastic optimization. Scenario tree based approaches (from now on denoted as SO, i.e., Stochastic Optimization) have been the subject of intense research in the last two decades; see e.g. 309 , Chapter 13] $59,202,235,330,331$ among the many other general references. Their use in the UC context has been considered e.g. in $74,289,367,405,411$. The key advantage of using scenario trees is that uncertainty is assumed to be known in each node of the tree. Since moreover uncertainty is now discretized on the tree, essentially this amounts to solving a deterministic UC of very large scale. The authors of 375] demonstrate the interest of SO over deterministic optimization using such a direct reformulation. According to [52, SO methods have two major drawbacks. First, obtaining an accurate probability distribution can be difficult, i.e., setting up an accurate tree is hard. Indeed, while generating scenarios for each individual uncertainty factor may be relatively straightforward, combining these to form a tree structure is not easy. Second, these solutions provide only probabilistic guarantees. The first difficulty can be partially tackled by the approaches considered in 121,123, 178 180], that provide a systematic approach for generating manageable trees. Classical approaches (e.g. [367|) to form a tree are those that start out with a set of scenarios and progressively regroup similar scenarios to form the nodes, in each of which a representing scenario is selected. The use of physical models for generating uncertainty (e.g. 95]) could also help improve the realism of the underlying scenario tree. The second 
difficulty can be tackled by using a hybrid approach that also considers spinning reserve requirements on the scenario tree 326 409], which can be used to account for events not modeled in the tree. We mention in passing that similar techniques can also be applied to longer-term problems, such as the management of an hydro reservoirs, that although not strictly pertinent to this paper are clearly strongly related. For a recent instance, a specialized stochastic dual DP algorithm is proposed in 170 .

4.1.1.2 Robust optimization. In order to be less demanding on the representation of uncertainty, Robust Optimization (RO) uses the notion of uncertainty set, which basically reunites the adverse events against which we wish to protect ourselves. For a comprehensive introduction to robust optimization we refer to 38, 51]; other important references are [40,42,53,54, 154, 155]. RO approaches might lead to a substantially higher costs of the proposed solution - a too high "price of robustness" [54] - w.r.t. SO ones when distributions of the uncertainty are sufficiently well characterized. This is mainly because RO protects against each event in the specified uncertainty set regardless of its probability, and therefore may have to account for extremely unlikely events. Several RO approaches have parameters (e.g., "budget of uncertainty") that can be used to adjust the degree of protection offered by the model [53, 84, 268]; yet, in general tuning these parameters is far from trivial. To reduce the price of robustness associated with classical ellipsoidal and $\Gamma$-robustness uncertainty sets proposed in $40,54,155$, subsequent studies have investigated alternative soft and light robustness models 37, 133. Recently, multiband robustness 69,70 , has been proposed as a generalization of $\Gamma$-robustness that can support an improved and stratified representation of uncertainty and a reduction in conservatism, while maintaining the computational tractability and accessibility of $\Gamma$-robustness.

4.1.1.3 Chance-Constrained Optimization. Chance-Constrained Optimization provides an attractive way to select the trade-off between cost and robustness, using a notion - the probability of the selected solution to be feasible - that is easy for the decision-maker to understand and manage. We refer to 110 309 310 for a modern introduction to probabilistic programming. In 381 the potentials for energy management applications, such as UC, are evaluated. However, a drawback of CCO is that probabilistic constraints can be nonconvex and hard to evaluate, thus making these approaches potentially computationally demanding.

4.1.1.4 The link between RO and CCO. There actually is an important link between RO and CCO. Indeed, an intuitively appealing idea is to select the uncertainty set in such a way as to enforce a probabilistic constraint, so that the solutions produced by the RO approach are comparable with those produced by the $\mathrm{CCO}$ one. More generally, one may aim at replacing the probabilistic constraint with a convex, albeit possibly more restrictive, constraint. There are various ways of doing this (e.g. 43,268$]$ ), often referred to as "safe-tractable approximation approaches" (a somewhat unfortunate terminology implicitly assuming that all CCO problems are intractable, which is not the case). Frequently, such convex outer approximations of the CCO-feasible set are derived by using individual probabilistic constraints, i.e., constraints that require that each individual inequality in the constraints system holds with high enough probability (e.g. 84 ). Besides using a (not necessarily very tight) approximation, this approach gives little control over the joint violation of the constraints, although it does have the advantage that convexity makes the corresponding problems easier to solve. We refer to 380,382 for examples showing that individual probabilistic constraints may lead to an arbitrary number of violated constraints. We also refer to 27,166 for various other alternatives of building uncertainty sets. The scenario approximation approach (e.g. 71, 267, 269]) can be seen as a special case of RO with a discrete uncertainty set that arose by drawing random samples from the underlying distribution.

\subsubsection{Modelling and solution choices}

4.1.2.1 The choice of recourse decisions. A crucial decision in all two-stage (or multi-stage) models, be they $\mathrm{SO}, \mathrm{RO}$ or $\mathrm{CCO}$, is which variables represent "here and now decisions" (first stage), to be taken 
before the uncertainty is revealed, and which represent "recourse actions" (second or later stages) that can change when the uncertain parameters are revealed. In multi-stage models a whole chain of decisions and observation of uncertainty needs to be worked out properly. This decision-observation chain may end with the observation of a last random realization offering no recourse actions. This could give rise to the need to consider multi-stage RO (CCO) approaches. When recourse is incomplete (i.e., can not guarantee feasibility of later stages regardless of the random realizations) such a need may also arise.

In general, recourse formulations aim at minimizing the total cost of the here and now decisions and the expected cost of the possible recourse actions. These problems are typically very challenging from both the computational and theoretical point of view, especially if recourse actions are integer-valued (or otherwise belong to a non-convex set). In the integer setting, a general approach to deal with this formulation was introduced by 211. In 234 a progressive hedging algorithm and Taboo search are used to address multi-stage problems with mixed $0-1$ variables. The approaches can become somewhat computationally less demanding if recourse variables are instead continuous, which is often the case in UC. In fact, here commitment variable are typically first-stage decisions, to be taken well in advance, while the actual energy production (usually continuous) is indeed managed in real time when the uncertain data (load, prices, ...) is revealed. Such a choice is made in 52 where RO is applied to UC with a 2 stage approach. Restricting commitment choices to a first stage is a convenient simplification but it does not fully represent reality, where (a few) changes to the commitment of units are in general possible. Accounting for recourse decisions, however, significantly increases the complexity of the problem, which justifies why restricting integer decisions to the first stage is the most common approach.

4.1.2.2 Direct approaches vs. decomposition. Regardless of the simplifying assumptions on UUC, the resulting mathematical program is frequently a very-large-scale one, which means that decomposition approaches are especially attractive. In some special situations, direct use of MI(N)LP solvers remains possible. This is for instance the case of the self-scheduling of a single unit subject to uncertain prices, for which the deterministic problem has a low number of variables. Often, however, the deterministic equivalent (if any) of the uncertain problem is usually so large that it cannot be directly solved by use of MILP solvers, and decomposition is required. This can be achieved by variable duplication, relaxing nonanticipativity constraints, system wide constraints or by using Benders' decomposition. The resulting sub-problems are then CCO (e.g. [379]), RO, deterministic (e.g. 367]) or stochastic programs (e.g. 74]).

We will now present more details on algorithms for Uncertain UC models using these three approaches.

\subsection{Stochastic Optimization (Scenario-Tree) approaches}

In this section we will discuss four common solution approaches to solving scenario-tree based versions of UC: the direct MILP approach and three decomposition methods.

A SO program with scenario-tree structure can be decomposed in at least two ways. Perhaps the most natural one is to relax the so-called non-anticipativity constraints and solve as many deterministic UC problems as there are scenarios. This is called the Scenario Decomposition approach 367] and includes well-known variants such as progressive hedging [321]. The alternative is to dualize the offer demand equilibrium constraints in each node to form a LD [74] and solve as many stochastic programming problems as there are units. This can be referred to as Space Decomposition, Unit Decomposition or Stochastic Decomposition, because one is basically optimizing a stochastic function, which in this case just happens to have an underlying discrete distribution. We will use Unit Decomposition, UD, to have a different shorthand from the Scenario Decomposition, SD. The discretization can be carried out after having formed the LD in an appropriate Banach space setting ( $L^{1}$-type spaces); see for instance 278]. We refer to [329] for a thorough discussion on various alternatives.

A different applicable approach is Benders' decomposition, cf. \$4.2.4. It exploits the $L$-shaped structure of the problem, whereby the second-stage (recourse) variables corresponding to each scenario are unrelated, and therefore the corresponding subproblems can be solved independently, once the first-stage variables 
are fixed 385. This corresponds to seeing the second (or later) stage(s) as an aggregated expected cost function depending on first (or earlier) stage variables. Under appropriate hypotheses (e.g., no integer decisions in later stages) this expected cost function can be shown to be convex, and cutting planes based approximations can then be used to compute the solution of the master problem (e.g. [108]).

\subsubsection{Mixed Integer Linear Programming}

In 377 the use of UC tools in a deregulated market is discussed. In particular, under the assumptions that prices are stochastic and there is no market power or transmission constraints, a GENCO can solve a self-scheduling UC for each of its units independently, which however should be a SO model due to uncertainty on prices. A MILP formulation for (a basic) UC is proposed, along with three DP approaches to solve it. These approaches are used to produce a cost-based method to generate a distribution of energy prices, based on the assumption that in a competitive market the price should be equal to the marginal cost of the most costly committed unit.

In 305 a two-stage model is considered where the first stage decisions consists of commitment decisions and an offer curve, while in the second stage the dispatch is computed. Single unit or identical unit systems are considered, although the model with several units can not cope with minimum up/down times. The focus is essentially on obtaining the offer-curve. A DP principle is presented, but no numerical experiments are provided. A very similar model is considered in 371, wherein commitment decisions and offer curves are first-stage decisions and dispatch later stage decisions. The key focus of these papers is on the market mechanisms.

Hydro scheduling is looked at in a market-based setting in [136]. The problem integrates commitment decisions on the turbined output, which have minimal release rates. Expected gain from selling energy on the market is maximized, whereas volume-dependent water values are used in order to represent the cost of water as measured by the difference between the initial and final volume in the reservoir.

The authors of 46 propose a two-stage formulation wherein the first stage variables consist of bilateral contracts. Once these contracts have been selected, the market price is observed and a bUC is solved in order to meet the resulting load. The objective function consists of Markovitz mean-variance model related to expected profits. A specialized B\&B method is used in order to solve the corresponding MILP problem; the numerical experiences cover a GENCO with 3 thermal units and up to 15 scenarios.

In 79 a weekly UC model is studied wherein profit of a GENCO depends on bids made on the market. The GENCO is assumed to have a non-linear non-convex effect on market prices, modeled through the use of piecewise linear functions and binary variables. The corresponding model is solved using a MILP solver, Lagrangian decomposition and two variants of Benders' decomposition (taken from [78]). The computed production schedule is a first stage decision, whereas all other stages and nodes in the scenario tree refer to different realizations of market settling. The Benders-based decomposition approaches are found to be the most interesting, despite the substantial implementation effort.

In 96 a two-stage model is considered where commitment decisions and bid prices are first-stage decisions, while total generation and energy matched in the day-ahead market are second-stage decisions (continuous variables). Uncertainty is mainly relative to the spot price, that enters in the generators objective function. The formulated MIQP has a quadratic second-stage cost function, which is linearized by means of perspective cuts 141 . The resulting problem with 10 scenarios and 9 thermal units is solved with a MIQP solver. In this vein we also cite 393, where the second stage economic dispatch problem, involving wind generation, is used for adding feasibility cuts to the first stage master problem. The main focus here is on deriving "robust" commitment decisions.

\subsubsection{Scenario Decomposition}

In 367. progressive hedging is used to solve a large-scale bUC with 100 thermal units and 6 hydro ones. A SD scheme is presented in 72,73 for solving a two-stage bUC problem (with only a few thermal 
units), wherein integer variables are restricted to the first stage. The non-anticipativity constraints are dualized by using Lagrangian multipliers, and the overall scheme is inserted into a B\&B procedure in order to ensure that an optimal solution is obtained. In 296 a scenario decomposition is used, with the focus being on reserve requirements in a system with high wind penetration. In 294] the uncertain renewable production is coupled with the demand response in a market environment. In [297] SD is again used to solve a UUC where the uncertainty is caused by wind power generation, taking into account the network constraints. A decomposition approach mixing scenario and Benders' decomposition is considered in 383. The investigated approach relies heavily on classical tools in deterministic UC, such as Lagrangian decomposition, Lagrangian-based primal recovery heuristics and Bundle methods, but needs no specific assumptions on the set of technically feasible schedules. A real-life problem with 136 thermal units, 22 hydro valleys, 96 times steps and 50 scenarios is solved.

\subsubsection{Unit (Stochastic) Decomposition}

The standard UD approach is proposed in 74 for a bUC with 50 thermal units; the demand constraints are relaxed, resulting in stochastic sub-problems which are then solved by DP.

In 324 a multi-stage hydro-thermal UC problem is considered with random customer load. The load is observed after having chosen the commitment decisions, but the actual generation levels (including continuous hydro generation) are determined once that the load is known. The demand constraint is dualized in a general probabilistic space setting, then the probability measure is discretized; no numerical results are presented.

A multi-stage stochastic programming is proposed in 277 to deal with a hydro-thermal UC with 25 thermal units and 7 hydro units. Load uncertainty is addressed through the use of UD and DP for solving the stochastic sub-problems; Lagrangian heuristics are then used to recover a primal solution. Similar UD approaches are considered in [111,161,276].

In 368, three uncertainty factors are integrated in the UC problem: load, fuel and electricity prices. The fuel requirement problem basically becomes the second stage of the problem, the first one being a bUC formulation. A Benders' decomposition approach is used to plug the second-stage cost function into the first stage, and a LR approach is used for the first stage. This method is tested on a UUC with 33 thermal units and about 729 demand scenarios.

In 21 a weekly (10 days up to a month) stochastic UC problem is considered. A UD approach is employed, where the LD is solved by a disaggregate Bundle method. The approach associates a set of weights with each node that effectively preconditions the LD; this preconditioning is reported to be crucial for performances. Problems having up to 2000 nodes are solved with the generating units of EDF.

A weekly two-stage UUC is also addressed in 342 . Both stages have all time steps, and essentially each is a bUC problem; load, price and cost uncertainty are revealed between the two. The problem is decomposed using a LR-based approach that yields a stochastic programming problem for each unit. Lagrangian heuristics based on 159,427 are employed to recover a primal feasible solution. The authors also present a MILP for market price settling and bidding in a competitive environment. They suggest to incorporate both features into a single model by moving bid/offer decisions and first day commitment decisions in a first stage, while all other variables are moved to the second stage. In 273 the authors consider a model, with focus on market mechanisms, wherein commitment decisions and offer curves are first-stage decisions and dispatch are later stage decisions. The authors apply a global LR-based UD for solving the thus formulated problem.

In 278 stochastic Lagrange multipliers are used in order to decompose uncertain demand constraints that have to hold almost surely. The resulting dual function is the expectation of this stochastic Lagrange function. Uncertainty is then discretized into a finite set of random drawings in order to approximate the expectation, and Bundle approaches are used to solve the dual. In this two-stage procedure, integer variables remain present in the second stage. 
In 354 the UD approach to the stochastic bUC with uncertain demand is revisited in terms of DantzigWolfe decomposition (the equivalence between this and a LR approach solved by CP being well-known). This results in a column generation approach where the Lagrangian subproblem, solved by DP on the scenario tree, generates schedules for each unit that are added to the restricted master problem.

\subsubsection{Benders(-Like) Decomposition}

The $L$-shaped method can be used to decompose UC problems with several stages. In its basic version a single cut is added to the first stage problem, whereas in advanced versions multiple cuts (e.g., one for each subproblem) can be added. This may increase convergence speed at the cost of an increased master problem cost; we refer to the discussion in [58,59 on this topic. The recent on-demand accuracy Bundle methods [106] can be thought to provide a tradeoff between the multi-cut and mono-cut versions 125].

In [412 another approach is proposed for finding such a trade-off. In this method, which is applied to a stochastic UC with load and generation uncertainty, scenarios are divided into (homogeneous) groups and cuts are derived for each group, as proposed in 372 . Consequently, the dimension of the master problem is smaller in comparison with the classical multi-cut algorithm, while less information is lost compared to the single cut version. The authors also claim that heterogeneously grouping the scenarios may result in even better CPU time. Results are presented for a large-scale thermal UC with ramp rates and spinning reserves.

In 14 short-term cascaded reservoir management - as opposed to the more traditional approach where reservoir management is considered to be a mid-term problem - is considered wherein the gain function is explicitly given and depends on the water level and turbined quantity. Uncertainty is modeled as a Markov chain having 6 states per time step, which is expanded onto a scenario tree in order to allow for an LP formulation of the problem. This approach is compared with DP, nested Benders' decomposition (closely related to SDDP) and a decomposed DP approach, which essentially efficiently samples the state space. Nested Benders' decomposition is found to be computationally the most efficient approach.

Benders' decomposition is compared with MILP approaches in [79] (cf. 4.2.1) and proves to be in general preferable. In 394, Benders' decomposition is used to address UC problems under wind uncertainty. The authors use sub-hourly time steps $(10,15$ or 30 minutes) to account for rapid variations in renewable generation. They also modify the standard approach by adding some of the second stage constraints to the master problem.

In 425 a two-stage UC formulation is considered. Similarly to most approaches load is revealed in between the first and second stage and power output is determined in the second stage, but the latter also contains integer commitment decisions related to quick-start units. The quadratic costs functions are linearized to obtain a MILP formulation. Then, because the second stage contains integer variables, the approach of 352 - essentially a Reformulation-Linearization-Techniques 351 with Lift-and-Project cuts [24 - is employed to construct an approximation of the convex hull of the second-stage problem, so that a multi-cut Benders approach can be used to approximate the second stage recourse cost function. A problem with 5 units, up to 2000 scenarios and 16 time steps is solved.

In 295 both LR and Benders' decomposition are used in a parallel high performance computing environment for solving a network constrained stochastic UC where uncertainty comes from different sources.

\subsection{Robust Optimization approaches}

An early work using RO techniques is 338, where a market clearing problem is considered under some UC-like constraints. The main idea is to use an adaptive $R O$ approach which partitions the uncertainty set and allows decisions to be specific to each subset. The constraints are then weighed in the master problem. The results are compared with traditional RO and a worst-case fully anticipative approach. 
In 400 a RO approach is considered where the uncertainty set on the load is a simple interval, so that methods from interval LP (e.g., [85]) can be employed together with Benders' decomposition to solve the model. The main focus of the work is on network security. In [410] a similar interval uncertainty approach is compared with a scenario-based approach. The results show that the former is very sensitive to the choice of the interval but is quickly solved, whereas the latter yields more accurate solutions but it is more costly to solve.

In 424 a 36 unit bUC with ramp rate constraints is considered which includes wind energy supply and demand behavior of the customers based on electricity prices. In this two-stage model, wind power enters under the guise of an uncertain budget constraint and the first stage is a day-ahead UC problem, while the second stage is performed once the wind supply is known. The problem is solved by applying Benders' decomposing to the linearized problem along with a $\mathrm{CP}$ algorithm. It is claimed that this model significantly reduces the total cost and can fully exploit the available supply of wind energy. The same approach is employed in 199 to solve a 30 unit UC with ramp rates and transmission constraints where demand and supply are considered to be uncertain.

In 52 the model proposed in 199,424 is extended to incorporate spinning reserve constraints, transmission limits and ramping constraints. The focus is on gauging the impact of robustness of the solutions on the efficiency and operational stability of the system. A two-stage adaptive RO model is used where the uncertainty set concerns the nodal net injection at each time period. In the first stage an optimal commitment decision is reached by using Benders' decomposition algorithm, while in the second stage the associated worst case dispatch cost is calculated. Results from empirical studies with 312 generators have been compared to those of deterministic models with reserve adjustments under three aspects: the average dispatch and total cost, the cost volatility, and the sensitivity of the costs to different probability distributions. The sensitivity of the results to changes in the uncertainty set is not investigated. A very simplified two-stage RO model is investigated in 36, where sensitivity to the choice of the uncertainty set is instead explicitly addressed. The recourse cost function is the worst case cost over a specific uncertainty set involving uncertainty on load; a simple recourse assumption makes the second stage trivial. In 250, 251 the model of 36 is expanded to take into account a huge uncertainty set which admits a representation as a "Markov chain". A budget of uncertainty constraint restricts paths to be "not too extreme"; a comparison is made against stochastic programming approaches.

The authors of 362 consider RO for uncertainty on contingency constraints. The resulting optimization problem is reformulated as an equivalent MILP and solved with standard solvers. This work is extended in 398 by including transmission capacity constraints and by considering a two-stage robust optimization setting. Commitment (and integer) variables are restricted to the first stage so that the second stage becomes a continuous optimization problem, further reduced to an LP by linearization techniques. A Bender's decomposition approach is used for solving the model. In 198 a similar model and solution approach can be found, integrating (interval) uncertainty on wind generation. A budget of uncertainty constraint limits conservativeness of the model. Demand response uncertainty is added in 423; the three stages of the model are brought down to two stages by a reformulation. Commitment decisions are restricted to the first stage and Bender's decomposition is again used for solving the problem. In 422 the authors add a convex combination of expected second stage cost and worst-case robust cost to the objective function. Uncertainty is restricted to load uncertainty and Bender's decomposition is employed for solving the model.

In [3] a RO approach to the management of electricity power generation is presented using concepts borrowed from classic risk management, i.e., Value-At-Risk. In [169] a RO with the Affinely Adjustable Robust Counterpart (AARC) approach 39 is proposed to the longer term electricity production management. AARC is a restricted and more tractable version of the Adjustable Robust Counterpart (ARC), where recourse variables are allowed to depend on the values of uncertain parameters, but only in an affine way. The same methods are looked at for weekly hydro reservoir management under uncertainty on inflows in 13 20. The hypotheses are set up in such a way that the resulting problem has a MILP deterministic equivalent, which is then solved by a MILP solver. Several comparisons with sliding deterministic approaches are presented. Finally, in 195 an adjustable robust OPF is suggested. 


\subsection{Chance-Constrained Optimization approaches}

In many optimization problems involving a final observation of uncertainty for which no recourse actions exist, one cannot guarantee feasibility for all constraints. Rather, one has to provide solutions which are "reasonably feasible" under all except the most unlikely scenarios. This is also the case in UC, where, for instance, one cannot actually guarantee that the demand constraints will never be violated. This is therefore an ideal setting for CCO, where the desired safety level can be specified under the form of a probability. Two approaches are possible: either the safety level is set for each constraint (e.g., time step) individually, giving an Individual CCO program, or for the system as a whole, resulting in a Joint CCO program. While the ICCO is obviously less robust than the JCCO (see the discussion in [382), the latter is in general significantly more difficult to solve, especially if one wishes to do this exactly (i.e., without artificially discretizing the underlying random vectors or approximating the probabilistic constraint). This explains why CCO (either Individual or Joint) models are the least employed in the literature on UC. However, it should be noted that these approaches have indeed been used in related problems such as power expansion and transmission ones 6, 347, 353, which need be formulated on a much longer time horizon than commonly considered in UC, and therefore crucially require taking uncertainty into account 353.

Individual CCO was applied for the first time in 289 to solve a 100-units bUC where the uncertainty of load has to be met with a high probability. The problem is then decomposed by using LR, and the subproblems are solved by DP. The results show that solving the CCO UC produces better (less costly) solutions than a deterministic UC with spinning reserves requirement.

In 116 a ICCO UC model is formulated where different sources of randomness are considered. In particular, demand fluctuation, thermal units outage, uncertainty of wind generation and the schedule of flexible generating units. The individual chance constraints are converted into a deterministic model using the central limit theorem to recover a Gaussian model of uncertainty for outages. A standard MILP approach is then used to solve the problem. Again, the results are compared with these of a deterministic UC formulation, and the authors claim that the proposed model could be extended to basically any stochastic factor.

A stylized UC model for hydro thermal systems under joint probabilistic constraints has been considered first in 429. The main focus there lies on dealing simultaneously with probabilistic constraints and binary variables, a significant technical feat. The suggested approach relies on the fact that some inequalities in the random system are more likely to be binding than others. This provides an ad-hoc way of reducing the difficulty for the JCCO (the experiments of 382 provide a rationale behind this approach). The reduced joint probabilistic constraint is then outer approximated by individual probabilistic constraints selecting appropriate weights. Finally, by using Hoeffding's inequality an outer and inner approximation of these latter individual probabilistic constraint can be obtained. The resulting binary conic programming problem can be solved with a standard solver.

In 397 a two-stage JCCO UC is considered with a joint probabilistic constraint for the use of wind power. The probabilistic constraint is not dealt with directly, but is discretized using a sample average approximation approach (e.g., 238,239$]$ ).

Joint probabilistic constraints in UC are dealt with exactly for the first time in 379. Two sources of uncertainty are considered: randomness on load and on inflows for hydro reservoirs. In order to solve the JCCO UC problem, various decomposition approaches are investigated, among which LR and various forms of AL approaches.

In 97] a DC Optimal Power Flow using an individual CCO approach is proposed considering the uncertainty of renewable generation. Under appropriate assumptions on the underlying distribution of uncertainty, and by reformulating the bilateral individual probabilistic constraints to two unilateral ones, the resulting problem can be shown to be equivalent to a second order cone problem. The conic constraints are then linearized by using a cutting planes approach. A real life instance over the 2746 bus Polish network is solved. It is interesting to note that such a network application with joint probabilistic constraints would give rise to differentiability issues, essential for the application of first-order methods; 
we refer to 183 for a thorough discussion of differentiability and an application to a stylized network problem.

Finally, it is worthwhile to note that stability theory for $\mathrm{CCO}$ is developed in [323] for recent references on such stability results we refer to 181, 182,184,322 and references therein. In particular, the authors explicitly consider stability results for probabilistically constrained power dispatch models, showing that the models are stable for several underlying distributions of the load, such as discrete or multi-variate Gaussian. However, no computational results are presented.

\section{Concluding Remarks}

The Unit Commitment problem could be considered an archetypal example of what makes optimization techniques both relevant and challenging.

UC regards the optimal use of a highly valuable resource, energy, whose importance has possibly never been more strongly felt than in the present times. On the one hand, energy is a primary driver of, and a necessary requirement for, economic growth and improvement of peoples' living conditions. On the other hand, fair and sustainable energy production and distribution raises enormous technical, economical, organizational, and even moral challenges. While optimization techniques (and in particular their strict subset regarding the UC problem) alone cannot clearly solve all these issues, they can indeed give a significant contribution to the improvement of the efficiency of the energy system, with a substantial positive economical and environmental impact.

From a technical perspective, UC arguably exhibits almost all possible characteristics that make an optimization problem extremely challenging. For a start it is not even a well-defined problem, but rather a large family of related problems that are as varied as the electrical systems worldwide. In almost all cases the problem is large- to very-large-scale, nonlinear, nonconvex and combinatorial. Thus, researchers continuously have to struggle between two contrasting needs: on the one hand providing more and more accurate models of the highly complex electrical systems, in order to allow better practical decisions, and on the other hand providing answers in the "unreasonably short" timeframe required by the actual operating environment. Furthermore, and perhaps more importantly for the present work, the operation of the electrical system requires a very articulate decision chain that spans from the decades (strategic decisions about the investments in new generation and transmission equipment, and even about funding of research capable of producing better ones) to the split-second range for on-line tracking of actual demand. This in turn means that uncertainty on the actual future status of the electrical system, and therefore on the consequences of the decisions that have to be taken here and now, is inherently present at all levels of the decision chain. This justifies the interest for techniques capable of dealing with uncertainty in energy optimization problems, and in particular in UC; whence the significance of this survey.

While UC cannot be presently considered a well-solved problem, and much less so UUC (which has arguably been tackled only relatively recently), research on such an extremely challenging problem will likely have positive side-effects. Indeed, the tools and techniques that will be developed will almost surely find applications in many different fields, other than the optimal management of the energy system. This has already happened for the methodological and algorithmic developments of $99,128,141,311$, that were motivated by the study of UC, but have since been applied to a much broader set of problems. We are confident that the study of UUC will lead, together with practical improvements on the efficiency and safety of electrical systems, to an analogous development of new ideas and techniques that will be beneficial for many other fields. Therefore, as a small stepping stone for researchers interested in broadening their knowledge in UUC, we hope that this survey may prove useful.

Acknowledgements The first author would like to thank Afsaneh Salari and Maryam Arbabzadeh for their input and their intellectual support. The second and third author gratefully acknowledge the support of the Gaspard Monge program for Optimization and Operations Research (PGMO) project "Consistent Dual Signals and Optimal Primal Solutions". 


$\begin{array}{ll}\text { UC } & \text { Unit-Commitment problem } \\ \text { UUC } & \text { UC problem under Uncertainty } \\ \text { bUC } & \text { basic UC problem (common modeling assumptions) } \\ \text { ED } & \text { Economic Dispatch } \\ \text { GENCO } & \text { GENeration COmpany } \\ \text { TSO } & \text { Transmission System Operator } \\ \text { MP } & \text { Monopolistic Producer } \\ \text { PE } & \text { Power Exchange } \\ \text { PEM } & \text { PE Manager } \\ \text { OTS } & \text { Optimal Transmission Switching } \\ \text { UCOTS } & \text { UC with OTS } \\ \text { MSG } & \text { Minimal Stable Generation } \\ \text { OPF } & \text { Optimal Power Flow } \\ \text { ROR } & \text { Run-Of-River hydro unit } \\ X_{1} & \text { set of technically feasible production schedules } \\ X_{2} & \text { set of system wide constraints } \\ \mathcal{T} & \text { set of time steps } \\ \text { MILP } & \text { Mixed-Integer Linear Programming } \\ \text { MIQP } & \text { Mixed-Integer Quadratic Programming } \\ \text { DP } & \text { Dynamic Programming } \\ \text { SDDP } & \text { Stochastic Dual DP } \\ \text { B\&B, B\&C, B\&P } & \text { Branch and Bound (Cut, Price respectively) } \\ \text { AL } & \text { Augmented Lagrangian } \\ \text { LR } & \text { Lagrangian Relaxation } \\ \text { LD } & \text { Lagrangian Dual } \\ \text { CP } & \text { Cutting Plane } \\ \text { SO } & \text { Stochastic Optimization } \\ \text { SD } & \text { Scenario Decomposition } \\ \text { UD } & \text { Unit Decomposition (also called space decomposition or stochastic decomposition) } \\ \text { RO } & \text { Robust Optimization } \\ \text { CCO } & \text { Chance-Constrained Optimization } \\ \text { ICCO } & \text { Chance-Constrained Optimization with Individual probabilistic constraints } \\ \text { JCCO } & \text { Chance-Constrained Optimization with Joint probabilistic constraints } \\ & \end{array}$

\section{References}

1. A. Y. Abdelaziz, M. Z. Kamh, S. F. Mekhamer, and M. A. L. Badr. An augmented hopfield neural network for optimal thermal unit commitment. International Journal of Power System Optimization, 2(1):37-49, June 2010.

2. M. Aganagic and S. Mokhtari. Security constrained economic dispatch using nonlinear dantzig-wolfe decomposition. IEEE Transactions on Power Systems, 12(1):105- 112, February 1997.

3. R. Aïd, V. Guigues, P.M. Ndiaye, F. Oustry, and F. Romanet. A value-at-risk approach for robust management of electricity power generation. Rapport de recherche, IMAG-LMC, 2006. submitted.

4. Y. Al-Kalaani, F.E. Villaseca, and F.Jr. Renovich. Storage and delivery constrained unit commitment. IEEE Transactions on Power Systems, 11(2):1059-1066, May 1996.

5. M. Amiri and S. Khanmohammadi. A primary unit commitment approach with a modification process. Applied Soft Computing, 13(2):1007-1015, February 2013.

6. G.J. Anders. Genetration planning model with reliability constraints. IEEE Transactions on Power Apparatus and Systems, PAS-100(12):4901-4908, December 1981.

7. E.J. Anderson and A.B. Philpott. Optimal offer construction in electricity markets. Mathematics of Operations Research, 27(1):82-100, 2002.

8. U.D. Annakkage, T. Numnonda, and N.C. Pahalawaththa. Unit commitment by parallel simulated annealing. IEE Proceedings-Generation, Transmission and Distribution, 142(6):595-600, November 1995.

9. L.T. Anstine, Burke R.E., J.E. Casey, R. Holgate, R.S. John, and H.G. Stewart. Application of probability methods to the determination of spinning reserve requirements for the pennsylvania-new jersey-maryland interconnection. IEEE Transactions on Power Apparatus and Systems, 82(68):726-735, October 1963. 
10. K.M. Anstreicher and L.A. Wolsey. Two "well-known" properties of subgradient optimization. Mathematical Programming, 120(1):213-220, 2009.

11. K. Aoki, M. Itoh, T. Satoh, K. Narah, and M. Kanezashi. Optimal long-term unit commitment in large scale systems including fuel constrained thermal and pumped storage hydro. IEEE Transactions on Power Systems, 4(3):1065-1073, August 1989.

12. K. Aoki, T. Satoh, M. Itoh, T. Ichimori, and K. Masegi. Unit commitment in a large-scale power system including fuel constrained thermal and pumped-storage hydro. IEEE Transactions on Power Systems, 2(4):1077- 1084, November 1987.

13. R. Apparigliato. Règles de décision pour la gestion du risque: Application á la gestion hebdomadaire de la production électrique. PhD thesis, École Polytechnique, Juin 2008.

14. T.W. Archibald, C.S. Buchanan, K.I.M. McKinnon, and L.C. Thomas. Nested benders decomposition and dynamic programming for reservoir optimisation. The Journal of the Operational Research Society, 50(5):468-479, 1999.

15. A. J. Ardakani and F. Bouffard. Identification of umbrella constraints in dc-based security-constrained optimal power flow. IEEE Transactions on Power Systems, 28(4):3924-3934, 2013.

16. J.M. Arroyo and A.J. Conejo. Optimal response of a thermal unit to an electricity spot market. IEEE Transactions on Power Systems, 15(3):1098-1104, 2000.

17. J.M. Arroyo and A.J. Conejo. Modeling of start-up and shut-down power trajectories of thermal units. IEEE Transactions on Power Systems, 19(3):1562-1568, 2004.

18. A. Astorino, A. Frangioni, M. Gaudioso, and E. Gorgone. Piecewise Quadratic Approximations in Convex Numerical Optimization. SIAM Journal on Optimization, 21(4):1418-1438, 2011.

19. H. Attouch, J. Bolte, P. Redont, and A. Soubeyran. Proximal alternating minimization and projection methods for nonconvex problems. an approach based on the kurdyka-lojasiewicz inequality. Mathematics of Operations Research, $35(2): 438-457,2010$.

20. F. Babonneau, J.P. Vial, and R. Apparigliato. Robust Optimization for Environmental and Energy Planning (Chapter 3 in [?]), volume 138 of International Series in Operations Research \& Management Science. Springer-Verlag, 2010.

21. L. Bacaud, C. Lemaréchal, A. Renaud, and C. Sagastizábal. Bundle methods in stochastic optimal power management: a disaggregate approach using preconditionners. Computation Optimization and Applications, 20(3):227-244, 2001.

22. L. Bahiense, N. Maculan, and C. Sagastizábal. The volume algorithm revisited: Relation with bundle methods. Mathematical Programming, 94(1):41-69, 2002.

23. A. Baillo, M. Ventosa, M. Rivier, and A. Ramos. Optimal offering strategies for generation companies operating in electricity spot markets. IEEE Transactions on Power Systems, 19(2):745-753, 2004.

24. E. Balas, S. Ceria, and G. Cornuéjols. A lift-and-project cutting plane algorithm for mixed 0-1 programs. Mathematical Programming, 58(1-3):295-324, 1993.

25. R. Baldick. The generalized unit commitment problem. IEEE Transactions on Power Systems, 10(1):465-475, 1995.

26. C. J. Baldwin, K. M. Dale, and R. F. Dittrich. A study of the economic shutdown of generating units in daily dispatch. Transactions of the American Institute of Electrical Engineers Power Apparatus and Systems, Part III, 78(4):1272- 1282, December 1959.

27. C. Bandi and D. Bertsimas. Tractable stochastic analysis in high dimensions via robust optimization. Mathematical Programming, 134(1):23-70, 2012.

28. F. Barahona and R. Anbil. The volume algorithm: Producing primal solutions with a subgradient method. Mathematical Programming, 87(3):385-399, 2000.

29. J.F. Bard. Short-term scheduling of thermal-electric generators using lagrangian relaxation. Operations Research, 36(5):765-766, 1988.

30. L. Baringo and A. J. Conejo. Offering strategy via robust optimization. IEEE Transactions on Power Systems, 26(3):1418-1425, 2011.

31. J. Batut and A. Renaud. Daily scheduling with transmission constraints: A new class of algorithms. IEEE Transactions on Power Systems, 7(3):982-989, 1992.

32. T.E. Bechert and H.G. Kwatny. On the optimal dynamic dispatch of real power. IEEE Transactions on Power Apparatus and Systems, PAS-91(1):889-898, 1972

33. R. E. Bellman and S.E. Dreyfus. Applied dynamic programming. Princeton University Press, New Jersey, 1962.

34. A. Belloni, A.L. Diniz, M.E. Maceira, and C. Sagastizábal. Bundle relaxation and primal recovery in unit-commitment problems. the brazilian case. Annals of Operations Research, 120(1-4):21-44, 2003.

35. C. Beltran and F.J. Heredia. Unit commitment by augmented lagrangian relaxation: Testing two decomposition approaches. Journal of Optimization Theory and Applications, 112(2):295-314, 2002.

36. S. Ben-Salem. Gestion Robuste de la production électrique à horizon court-terme. PhD thesis, Ecole Centrale Paris, Mars 2011.

37. A. Ben-Tal, D. Bertsimas, and D. Brown. A soft robust model for optimization under ambiguity. Operations Research, 58(4):1220-1234, 2010.

38. A. Ben-Tal, L. El Ghaoui, and A. Nemirovski. Robust Optimization. Princeton University Press, 2009.

39. A. Ben-Tal, A. Goryashko, E. Guslitzer, and A. Nemirovski. Adjustable robust counterpart of uncertain linear programs. Mathematical Programming, series A, 99:351376, 2003.

40. A. Ben-Tal and A. Nemirovski. Robust convex optimization. Mathematics of Operations Research, 23(4):769-805, November 1998

41. A. Ben-Tal and A. Nemirovski. Robust solutions of uncertain linear programs. Operations Research Letters, 25(1):113, August 1999.

42. A. Ben-Tal and A. Nemirovski. Robust solutions of linear programming problems contaminated with uncertain data. Mathematical Programming, series A, 88:411-424, 2000.

43. A. Ben-Tal and A. Nemirovski. On safe tractable approximations of chance-constrained linear matrix inequalities. Mathematics of Operations Research, 34(1):1-25, 2009. 
44. J.F. Benders. Partitioning procedures for solving mixed-variables programming problems. Numerische Mathematik, 4(1):238-252, 1962

45. F.E. Benth, R. Kiesel, and A. Nazarova. A critical empirical study of three electricity spot price models. Energy Economics, 34(5):1589-1616, September 2012.

46. P. Beraldi, D. Conforti, and A. Violi. A two-stage stochastic programming model for electric energy producers. Computers E Operations Research, 35:3360-3370, 2008.

47. D. Bertsekas, G. Lauer, N.R. Sandell-Jr, and T.A. Posbergh. Optimal short-term scheduling of large-scale power systems. IEEE Transactions on Automatic Control, 28(1):1-11, January 1983

48. D.P. Bertsekas. Nonlinear Programming. Athena Scientific, 2nd edition, 1999.

49. D.P. Bertsekas. Dynamic Programming \& Optimal Control, Vol I. Athena Scientific, 3rd edition, 2005.

50. D.P. Bertsekas. Dynamic Programming \& Optimal Control, Vol II: Approximate Dynamic Programming. Athena Scientific, 4th edition, 2012.

51. D. Bertsimas, D. Brown, and C. Caramanis. Theory and applications of robust optimization. SIAM Review, 53(3):464$501,2011$.

52. D. Bertsimas, E. Litvinov, X. A. Sun, J. Zhao, and T. Zheng. Adaptive robust optimization for the security constrained unit commitment problem. IEEE Transactions on Power Systems, 28(1):52-63, March 2013.

53. D. Bertsimas and M. Sim. Robust discrete optimization and network flows. Mathematical Programming, 98(1):49-71, September 2003

54. D. Bertsimas and M. Sim. The price of robustness. Operations Research, 52(1):35-53, February 2004.

55. D. Bienstock. Progress on solving power flow problems. Optima, 93:1-8, 2013.

56. D. Bienstock and A. Verma. The $\mathrm{n}-\mathrm{k}$ problem in power grids: New models, formulations and numerical experiments. Siam J. on Optimization, 2011.

57. R. Billinton and R. Karki. Capacity reserve assessment using system well-being analysis. IEEE Transactions on Power Systems, 14(2):433- 438, May 1999.

58. J. R. Birge and F. Louveaux. A multicut algorithm for two-stage stochastic linear programs. European Journal of Operational Research, 34(3):384-392, 1988.

59. J.R. Birge and F. Louveaux. Introduction to Stochastic Programming. Springer, New York, 1997.

60. E. Bompard and Y. Ma. Models of strategic bidding in electricity markets under network constraints. In A. Sorokin, S. Rebennack, P.M. Pardalos, N.A. Iliadis, and M.V.F. Pereira, editors, Handbook of Networks in Power Systems I, pages 3-39. Springer Heidelberg Berlin, 2012.

61. S.D. Bond and B. Fox. Optimal thermal unit scheduling using improved dynamic programming algorithm. IEEE Proceedings C, 133(1):1-5, January 1986.

62. J.F. Bonnans, J.C. Gilbert, C. Lemaréchal, and C. Sagastizábal. Numerical Optimization: Theoretical and Practical Aspects. Springer-Verlag, 2nd edition, 2006.

63. A. Borghetti, C. D'Ambrosio, A. Lodi, and S. Martello. A MILP approach for short-term hydro scheduling and unit commitment with head-dependent reservoir. IEEE Transactions on Power Systems, 23(3):1115-1124, 2008.

64. A. Borghetti, A. Frangioni, F. Lacalandra, A. Lodi, S. Martello, C.A. Nucci, and A. Trebbi. Lagrangian relaxation and tabu search approaches for the unit commitment problem. In IEEE Power Tech Proceedings, 2001 Porto, volume 3 , 2001 .

65. A. Borghetti, A. Frangioni, F. Lacalandra, and C.A. Nucci. Lagrangian heuristics based on disaggregated bundle methods for hydrothermal unit commitment. IEEE Transactions on Power Systems, 18:313-323, 2003.

66. A. Borghetti, A. Frangioni, F. Lacalandra, C.A. Nucci, and P. Pelacchi. Using of a cost-based unit commitment algorithm to assist bidding strategy decisions. In A. Borghetti, C.A. Nucci, and M. Paolone, editors, Proceedings IEEE 2003 Powertech Bologna Conference, volume Paper n. 547, 2003

67. F. Bouffard and F.D. Galiana. Stochastic security for operations planning with significant wind power generation. IEEE Transactions on Power Systems, 23(2):306-316, May 2008.

68. O. Briant, C. Lemaréchal, Ph. Meurdesoif, S. Michel, N. Perrot, and F. Vanderbeck. Comparison of bundle and classical column generation. Mathematical Programming, 113(2):299-344, 2008.

69. C. Büsing and F. D'Andreagiovanni. New results about multi-band uncertainty in robust optimization. In R. Klasing, editor, Experimental Algorithms - SEA 2012, volume 7276 of LNCS, pages 63-74, 2012.

70. C. Büsing and F. D'Andreagiovanni. Robust optimization under multi-band uncertainty - part i: Theory. Technical Report ZIB-Report 13-10, Zuse-Institut Berlin (ZIB), 2013.

71. G. C. Calafiore and M. C. Campi. Uncertain convex programs: Randomized solutions and confidence levels. Mathematical Programming, 102(1):25-46, 2005.

72. C.C. Carøe, A. Ruszczyński, and R. Schultz. Unit commitment under uncertainty via two-stage stochastic programming. In Proceedings of NOAS 1997, 1997.

73. C.C. Carøe and R. Schultz. A two-stage stochastic program for unit-commitment under uncertainty in a hydro-thermal power system. Technical report, ZIB, 1998.

74. P. Carpentier, G. Cohen, J. C. Culioli, and A. Renaud. Stochastic optimization of unit commitment: a new decomposition framework. IEEE Transactions on Power Systems, 11(2), may 1996.

75. M. Carrión and J.M. Arroyo. A computationally efficient mixed-integer linear formulation for the thermal unit commitment problem. IEEE Transactions on Power Systems, 21(3):1371-1378, August 2006.

76. J. P. S. Catalão, S. J. P. S. Mariano, V. M. F. Mendes, and L. A. F. M. Ferreira. Parameterisation effect on the behavior of a head-dependent hydro chain using a nonlinear model. Electric Power Systems Research, 76:404-412 2006.

77. J. P. S. Catalão, S.J.P.S. Mariano, V. M. F. Mendes, and L. A. F. M. Ferreira. Nonlinear optimization method for short-term hydro scheduling considering head-dependency. European Transactions on Electrical Power, 20:172-183, 2010 . 
78. S. Cerisola. Benders decomposition for mixed integer problems: Application to a medium term hydrothermal coordination problem. PhD thesis, Instituto Investigación Tecnológica Madrid, 2004.

79. S. Cerisola, A. Baíllo, J.M. Fernández-López, A. Ramos, and R. Gollmer. Stochastic power generation unit commitment in electricity markets: A novel formulation and a comparison of solution methods. Operations Research, $57(1): 32-46,2009$

80. M. Cerjan, D. Marcic, and M. Delimar. Short term power system planning with water value and energy trade optimisation. In International Conference on the European Energy Market (EEM), 2011.

81. K. Chandrasekaran and S. P. Simon. Multi-objective scheduling problem: Hybrid approach using fuzzy assisted cuckoo search algorithm. Swarm and Evolutionary Computation, 5:1-12, 2012.

82. K. Chandrasekaran and S. P. Simon. Network and reliability constrained unit commitment problem using binary real coded firefly algorithm. Electrical Power and Energy Systems, 43:921-932, 2012.

83. G.W. Chang, M. Aganagic, J.G. Waight, J. Medina, T. Burton, S. Reeves, and M. Christoforidis. Experiences with mixed integer linear programming based approaches on short-term hydro scheduling. IEEE Transactions on Power systems, 16(4):743-749, 2001.

84. X. Chen, M. Sim, and P. Sun. A robust optimization perspective on stochastic programming. Operations Research, 55(6):1058-1071, 2007.

85. J.W. Chinneck and K. Ramadan. Linear programming with interval coefficients. The Journal of the Operational Research Society, 51(2):209-220, May 2000.

86. S. Chitra-Selvi, R.P. Kumundi-Devi, and C.C. Asir-Rajan. Hybrid evolutionary programming approach to multi-area unit commitment with import and export constraints. International Journal of Recent Trends in Engineering, 1(3), May 2009.

87. A.I. Cohen and S. H. Wan. A method for solving the fuel constrained unit commitment problem. IEEE Transactions on Power Systems, 2(3):608-614, August 1987.

88. A.I. Cohen and M. Yoshimura. A branch-and-bound algorithm for unit commitment. IEEE Transactions on Power Apparatus and Systems, 102(2):444-451, February 1983.

89. G. Cohen. Auxiliairy problem principle and decomposition of optimization problems. Journal of optimization Theory and Applications, 32(3):277-305, 1980.

90. G. Cohen and D.L. Zhu. Decomposition-coordination methods in large-scale optimization problems. the nondifferentiable case and the use of augmented Lagrangians. Large Scale Systems, Theory and Applications, $1,1983$.

91. A.J. Conejo, M. Carrión, and J.M. Morales. Decision Making Under Uncertainty in Electricity Markets, volume 153 of International Series in Operations Research \& Management Science. Springer, 1st edition, 2010.

92. A.J. Conejo, J. Contreras, J.M. Arroyo, and S. de la Torre. Optimal response of an oligopolistic generating company to a competitive pool-based electric power market. IEEE Transactions on Power Systems, 17(2):424-430, 2002.

93. A.J. Conejo, F.J. Nogales, and J.M. Arroyo. Price-taker bidding strategy under price uncertainty. IEEE Transactions on Power Systems, 17(4):1081-1088, 2002.

94. A.J. Conejo and F.J. Prieto. Mathematical programming and electricity markets. TOP, 9(1):1-53, 2001.

95. E.M. Constantinescu, V.M. Zavala, M. Rocklin, S. Lee, and M. Anitescu. A computational framework for uncertainty quantification and stochastic optimization in unit commitment with wind power generation. IEEE Transactions on Power Systems, 26(1):431-441, 2011.

96. C. Corchero, E. Mijangos, and F.-J. Heredia. A new optimal electricity market bid model solved through perspective cuts. TOP, 21(1):84-108, 2013.

97. M. Chertkov D. Bienstock and S. Harnett. Chance-constrained dc-opf. working paper, submitted, 2014.

98. C. d'Ambrosio, A. Lodi, and S. Martello. Piecewise linear approxmation of functions of two variables in MILP models. Operations Research Letters, 38:39-46, 2010.

99. A. Daniildis and C. Lemaréchal. On a primal-proximal heuristic in discrete optimization. Math. Programming Series A, 104:105-128, 2005.

100. G. d'Antonio and A. Frangioni. Convergence Analysis of Deflected Conditional Approximate Subgradient Methods. SIAM Journal on Optimization, 20(1):357-386, 2009.

101. G.B. Dantzig and P. Wolfe. The decomposition principle for linear programs. Operations Research, 8:101-111, 1960.

102. D. Dasgupta and D.R. McGregor. Thermal unit commitment using genetic algorithms. IEEE Proceedings-Generation, Transmission and Distribution, 141(5):459- 465, September 1994.

103. A.K. David and F. Wen. Strategic bidding in competitive electricity markets: a literature survey. In Proceedings IEEE PES Summer Meeting, volume 4, pages 2168-2173, 2001.

104. D. P. de Farias and B. Van Roy. The linear programming approach to approximate dynamic programming. Operations Research, 51(6):850-865, September 2003.

105. S. de la Torre, J.M. Arroyo, A.J. Conejo, and J. Contreras. Price maker self-scheduling in a pool-based electricity market: A mixed-integer LP approach. IEEE Transactions on Power Systems, 17(4):1037-1042, November 2002.

106. W. de Oliveira and C. Sagastizábal. Level bundle methods for oracles with on demand accuracy. To appear in Optimization Methods and Software, pages 1-31, 2014.

107. W. de Oliveira, C. Sagastizábal, and C. Lemaréchal. Bundle methods in depth: a unified analysis for inexact oracles. Available at http://www.optimization-online.org/DB_HTML/2013/02/3792.html, 2013.

108. W. de Oliveira, C.A. Sagastizábal, and S. Scheimberg. Inexact bundle methods for two-stage stochastic programming. SIAM Journal on Optimization, 21(2):517-544, 2011.

109. G. Demartini, T.R. De Simone, G.P. Granelli, M. Montagna, and K. Robo. Dual programming methods for large-scale thermal generation scheduling. IEEE Transactions on Power Systems, 13:857-863, 1998.

110. D. Dentcheva. Optimisation Models with Probabilistic Constraints. Chapter 4 in [?]. MPS-SIAM series on optimization. SIAM and MPS, Philadelphia, 2009. 
111. D. Dentcheva and W. Römisch. Optimal power generation under uncertainty via stochastic programming. In K. Marti and P. Kall, editors, Stochastic Programming Methods and Technical Applications, volume 458 of Lecture Notes in Economics and Mathematical Systems, pages 22-56. Springer Berlin Heidelberg, 1998.

112. Cour des Comptes. Les coûts de la filière électronucléaire. Technical report, Cour des Comptes, 2012.

113. V.N. Dieu and W. Ongsakul. Ramp rate constrained unit commitment by improved priority list and augmented lagrange hopfield network. Electric Power Systems Research, 78(3):291-301, March 2008.

114. T. S. Dillon, K. W. Edwin, H.D. Kochs, and R. J. Taud. Integer programming approach to the problem of optimal unit commitment with probabilistic reserve determination. IEEE Transactions on Power Apparatus and Systems, PAS-97(6):2154- 2166, November 1978

115. T.S. Dillon and G.T. Egan. The application of combinatorial methods to the problems of maintenance scheduling and unit commitment in large power system. In 1st IFAC Symposium on Large Scale Systems Theory and Applications, udine, Italy, 1976.

116. X. Ding, W-J. Lee, W. Jianxue, and L. Liu. Studies on stochastic unit commitment formulation with flexible generating units. Electric Power Systems Research, 80:130-141, 2010.

117. A.K. Diongue. Modélisation longue mémoire multivariée : applications aux problématiques du producteur d'EDF dans le cadre de la libéralisation du marché européen de l'électricité. PhD thesis, ENS Cachan, 2005.

118. O. du Merle, J.-L. Goffin, and J.-P. Vial. On improvements to the analytic center cutting plane method. Computational Optimization and Applications, 11:37-52, 1998.

119. L. Dubost, R. Gonzalez, and C. Lemaréchal. A primal-proximal heuristic applied to french unitcommitment problem. Mathematical programming, 104(1):129-151, 2005.

120. H. Duo, H. Sasaki, T. Nagata, and H. Fujita. A solution for unit commitment using lagrangian relaxation combined with evolutionary programming. Electric Power Systems Research, 51(1):71-77, July 1999.

121. J. Dupačová, N. Gröwe-Kuska, and W. Römisch. Scenario reduction in stochastic programming : An approach using probability metrics. Mathematical Programming, 95(3):493-511, March 2003.

122. K. Ea. The electricity spot markets prices modeling: Proposal for a new mathematical formulation taking into account the market player strategy. In International Conference on the European Energy Market (EEM), 2012.

123. A. Eichhorn, H. Heitsch, and W. Römisch. Stochastic optimization of electricity portfolios: Scenario tree modeling and risk management. In S. Rebennack, P.M. Pardalos, M.V.F. Pereira, and N. Iliadis, editors, Handbook of Power Systems II, pages 405-432. Springer-Verlag, 2010

124. I. Erkmen and B. Karatas. Short-term hydrothermal coordination by using multi-pass dynamic programming with successive approximation. In 7th Mediterranean Electrotechnical Conference 1994, volume 3, pages 925-928, 1994.

125. C.I. Fábián. Computational aspects of risk-averse optimisation in two-stage stochastic models. Technical report, Institute of Informatics, Kecskemét College, Hungary, 2013. Optimization Online report.

126. W. Fan, X. Guan, and Q. Zhai. A new method for unit commitment with ramping constraints. Electric Power Systems Research, 62(3):215-224, July 2002.

127. I.A. Farhat and M.E. El-Hawary. Optimization methods applied for solving the short-term hydrothermal coordination problem. Electric Power Systems Research, 79:1308-1320, 2009.

128. S. Feltenmark and K.C. Kiwiel. Dual applications of proximal bundle methods, including lagrangian relaxation of nonconvex problems. SIAM Journal on Optimization, 10(3):697-721, 2000.

129. L.A.F.M. Ferreira. On the convergence of the classic hydro-thermal coordination algorithm. IEEE Transactions on Power Systems, 9:1002-1008, 1994.

130. E.C. Finardi and M.R. Scuzziato. Hydro unit commitment and loading problem for day-ahead operation planning problem. Electrical Power and Energy Systems, 44:7-16, 2013.

131. E.C. Finardi and M.R. Scuzziato. A comparative analysis of different dual problems in the lagrangian relaxation context for solving the hydro unit commitment problem. Electric Power Systems Research, 107:221-229, 2014.

132. E.C. Finardi and E.L. Da Silva. Solving the hydro unit commitment problem via dual decomposition and sequential quadratic programming. IEEE Transactions on Power Systems, 21(2):835-844, 2006.

133. M. Fischetti and M. Monaci. Light robustness. In R.K. Ahuja, R. Möhring, and C. Zaroliagis, editors, Robust and Online Large-Scale Optimization, volume 5868 of LNCS, pages 61-84, 2009.

134. E.B. Fisher, R.P. O'Neill, and M.C. Ferris. Optimal transmission switching. IEEE Transactions on Power Systems, 23(3):1346-1355, 2008 .

135. M.L. Fisher. Optimal solution of scheduling problems using lagrange multipliers: Part i. Operations Research, 21(5):1114-1127, October 1973.

136. S.-E. Fleten and T.K. Kristoffersen. Short-term hydropower production planning by stochastic programming. Computers \& Operations Research, 35:2656-2671, 2008.

137. M. Fonoberova. Algorithms for finding optimal flows in dynamic networks. In S. Rebennack, P.M. Pardalos, M.V.F. Pereira, and N. Iliadis, editors, Handbook of Power Systems II, pages 31-54. Springer, 2010.

138. M. Fotuhi-Firuzabad and R. Billinton. A reliability framework for generating unit commitment. Electric Power Systems Research, 56(1):81-88, October 2000.

139. A. Frangioni. Generalized bundle methods. SIAM Journal on Optimization, 13(1):117-156, 2002

140. A. Frangioni. About Lagrangian Methods in Integer Optimization. Annals of Operations Research, 139(1):163-193, 2005 .

141. A. Frangioni and C. Gentile. Perspective Cuts for a Class of Convex 0-1 Mixed Integer Programs. Mathematical Programming, 106(2):225-236, 2006

142. A. Frangioni and C. Gentile. Solving non-linear single-unit commitment problems with ramping constraints. Operations Research, 54(4):767-775, 2006

143. A. Frangioni, C. Gentile, and F. Lacalandra. Solving Unit Commitment Problems with General Ramp Contraints. International Journal of Electrical Power and Energy Systems, 30:316-326, 2008. 
144. A. Frangioni, C. Gentile, and F. Lacalandra. Tighter Approximated MILP Formulations for Unit Commitment Problems. IEEE Transactions on Power Systems, 24(1):105-113, 2009.

145. A. Frangioni, C. Gentile, and F. Lacalandra. Sequential Lagrangian-MILP Approaches for Unit Commitment Problems. International Journal of Electrical Power and Energy Systems, 33:585-593, 2011.

146. Y. Fu, Z. Li, and L. Wu. Modeling and solution of the large-scale security-constrained unit commitment. IEEE Transactions on Power Systems, 28(4):3524-3533, 2013.

147. Y. Fu and M. Shahidehpour. Fast SCUC for large-scale power systems. IEEE Transactions on Power Systems, 22(4):2144-2151, November 2007.

148. Y. Fu, M. Shahidehpour, and Z. Li. Long-term security-constrained unit commitment: hybrid dantzig-wolfe decomposition and subgradient approach. IEEE Transactions on Power Systems, 20(4):2093-2106, November 2005.

149. S.A. Gabriel, A.J. Conejo, J.D. Fuller, B.F. Hobbs, and C. Ruiz. Complementarity Modeling in Energy Markets, volume 180 of International Series in Operations Research E Management Science. Springer, 1 st edition, 2013.

150. J. García-González, A.M. San Roque, F.A. Campos, and J. Villar. Connecting the intraday energy and reserve markets by an optimal redispatch. IEEE Transactions on Power Systems, 22(4):2220-2231, 2007.

151. L. L. Garver. Power generation scheduling by integer programming-development of theory. Transactions of the American Institute of Electrical Engineers Power Apparatus and Systems, Part III, 81(3):730- 734, April 1962.

152. W. Ge. Ramp rate constrained unit commitment by improved priority list and enhanced particle swarm optimization. In International Conference on Computational Intelligence and Software Engineering (CiSE), 2010, pages 1-8, 2010.

153. D. Georges. Optimal unit commitment in simulations of hydrothermal power systems: an augmented lagrangian approach. Simulation Practice and Theory, 1(4):155-172, March 1994.

154. L. El Ghaoui and H. Lebret. Robust solutions to least-squares problems with uncertain data. SIAM Journal on Matrix Analysis and Applications, 18(4):1035-1064, July 2006.

155. L. El Ghaoui, F. Oustry, and H. Lebret. Robust solutions to uncertain semidefinite programs. SIAM Journal on Optimization, 9(1):33-52, 1998.

156. H. A. Gil, C. Gómez-Quiles, A. Gómez-Exposito, and J. R. Santos. Forecasting prices in electricity markets: Needs, tools and limitations. In A. Sorokin, S. Rebennack, P.M. Pardalos, N.A. Iliadis, and M.V.F. Pereira, editors, Handbook of Networks in Power Systems I, pages 123-150. Springer Heidelberg Berlin, 2012.

157. P.E. Gill, W. Murray, and M.H. Wright. Practical Optimization. Emerald Group Publishing Limited, London, 1st edition, 1982 .

158. T. Gjengedal. Emission constrained unit-commitment (ECUC). IEEE Transaction on Energy Conversion, 11(1):132138, March 1996.

159. R. Gollmer, A. Moller, M.P. Nowak, W. Romisch, and R. Schultz. Primal and dual methods for unit commitment in a hydro-thermal power system. In Proceedings 13th Power Systems Computation Conference, pages 724-730, 1999.

160. H.B. Gooi, D.P. Mendes, K.R.W. Bell, and D.S. Kirschen. Optimal scheduling of spinning reserve. IEEE Transactions on Power Systems, 14(4):1485- 1492, November 1999.

161. N. Gröwe-Kuska, K.C. Kiwiel, M.P. Nowak, W. Römisch, and I. Wegner. Power management in a hydro-thermal system under uncertainty by lagrangian relaxation. In C. Greengard and A. Ruszczyński, editors, Decision Making Under Uncertainty, volume 128 of The IMA Volumes in Mathematics and its Applications, pages 39-70. Springer New York, 2002.

162. X. Guan, P. B. Luh, and L. Zhang. Nonlinear approximation method in lagrangian relaxation-based algorithms for hydrothermal scheduling. IEEE Transactions on Power Systems, 10:772-778, 1995.

163. X. Guan, P.B. Luh, Y. Houzhong, and J.A. Amalfi. Environmentally constrained unit commitment. In Power Industry Computer Application Conference, Baltimore, MD, 1991.

164. X. Guan, P.B. Luh, H. Yan, and P. Rogan. Optimization-based scheduling of hydrothermal power systems with pumped-storage units. IEEE Transactions on Power Systems, 9:1023-1031, 1994.

165. X. Guan, E. Ni, R. Li, and P.B. Luh. An optimization-based algorithm for scheduling hydrothermal power systems with cascaded reservoirs and discrete hydro constraints. IEEE Transactions on Power Systems, 12:1775-1780, 1997.

166. Y. Guan and J. Wang. Uncertainty sets for robust unit commitment. IEEE Transactions on Power Systems, 29(3):1439-1440, 2014.

167. M. Guignard. Lagrangean relaxation. TOP, 11(2):151-228, 2003.

168. M. Guignard and S. Kim. Lagrangian decomposition: a model yielding stronger lagrangian bounds. Mathematical Programming, 39:215-228, 1987.

169. V. Guigues. Robust product management. Optimization and Engineering, 10(4):505-532, 2009.

170. V. Guigues. SDDP for some interstage dependent risk-averse problems and application to hydro-thermal planning. Computational Optimization and Applications, 10(4):505-532, 2013.

171. H. Habibollahzadeh and J. A. Bubenko. Application of decomposition techniques to short-term operation planning of hydrothermal power system. IEEE Transactions on Power Systems, 1(1):41-47, February 1986.

172. K. Hara, M. Kimura, and N. Honda. A method for planning economic unit commitment and maintenance of thermal power systems. IEEE Transactions on Power Apparatus and Systems, PAS-85(5):427-436, May 1966.

173. C. Harris. Electricity markets: Pricing, structures and Economics, volume 565 of The Wiley Finance Series. John Wiley and Sons, 2011.

174. K.W. Hedman, M.C. Ferris, R.P. O'Neill, E.B. Fisher, and S.S. Oren. Co-optimization of generation unit commitment and transmission switching with n-1 reliability. IEEE Transactions on Power Systems, 25(2):1052-1063, 2010.

175. K.W. Hedman, R.P. O'Neill, E.B. Fisher, and S.S. Oren. Optimal transmission switching with contingency analysis. IEEE Transactions on Power Systems, 24(3):1577-1586, 2009.

176. K.W. Hedman, S.S. Oren, and R.P. O'Neill. Optimal transmission switching: economic efficiency and market implications. Journal of Regulatory Economics, 40(3):111-140, 2011.

177. K.W. Hedman, S.S. Oren, and R.P. O'Neill. A review of transmission switching and network topology optimization. In Power and Energy Society General Meeting, 2011 IEEE, pages 1-7. IEEE, 2011. 
178. H. Heitsch and W. Römisch. Scenario reduction algorithms in stochastic programming. Computation Optimization and Applications, 24(2-3):187-206, 2003.

179. H. Heitsch and W. Römisch. Scenario tree reduction for multistage stochastic programs. Computational Management Science, 6(2):117-133, May 2009.

180. H. Heitsch and W. Römisch. Scenario tree generation for multi-stage stochastic programs. In M. Bertocchi, G. Consigli, and M.A.H. Dempster, editors, Stochastic Optimization Methods in Finance and Energy: New Financial Products and Energy Market Strategies, volume 163 of International Series in Operations Research 8 Management Science, pages 313-341. Springer-Verlag, 2011.

181. R. Henrion, C. Küchler, and W. Römisch. Discrepancy distances and scenario reduction in two-stage stochastic integer programming. Journal of Industrial and Management Optimization, 4:363-384, 2008.

182. R. Henrion, C. Küchler, and W. Römisch. Scenario reduction in stochastic programming with respect to discrepancy distances. Computational Optimization and Applications, 43:67-93, 2009.

183. R. Henrion and A. Möller. A gradient formula for linear chance constraints under Gaussian distribution. Mathematics of Operations Research, 37:475-488, 2012.

184. R. Henrion and W. Römisch. Hölder and lipschitz stability of solution sets in programs with probabilistic constraints. Mathematical Programming, 100:589-611, 2004.

185. F.J. Heredia and N. Nabona. Optimum short-term hydrothermal scheduling with spinning reserve through network flows. IEEE Transactions on Power Systems, 10:1642-1651, 1995.

186. H. Higgs and A. Worthington. Stochastic price modeling of high volatility, mean-reverting, spike-prone commodities: The australian wholesale spot electricity market. Energy Economics, 30(6):3172-3185, November 2008.

187. H.L. Hijazi, C. Coffrin, and P. Van Hentenryck. Convex quadratic relaxations of nonlinear programs in power systems. submitted, 2013.

188. B.F. Hobbs, M. Rothkopf, R.P. O'Neill, and H.P. Chao. The next generation of Electric Power unit commitment models. Number 36 in International Series in Operations Research \& Management Science. Springer, 2001.

189. W.J. Hobbs, G. Hermon, S. Warner, and G.B. Shelbe. An enhanced dynamic programming approach for unit commitment. IEEE Transactions on Power Systems, 3(3):1201-1205, August 1988.

190. Y.Y. Hsu, C-C. Su, C-J Lin, and C-T Huang. Dynamic security constrained multi-area unit commitment. IEEE Transactions on Power Systems, 6(3):1049-1055, August 1991.

191. K.Y. Huang, H.T. Yang, and C.L. Huang. A new thermal unit commitment approach using constraint logic programming. IEEE Transactions on Power Systems, 13(3):936- 945, August 1998.

192. R. A. Jabr. Radial distribution load flow using conic programming. IEEE Transactions on Power Systems, 21(3):1458-1459, 2006.

193. R. A. Jabr. Optimal power flow using an extended conic quadratic formulation. IEEE Transactions on Power Systems, 23(3):1000-1008, 2008 .

194. R. A. Jabr. Recent developments in optimal power flow modeling. In S. Rebennack, P.M. Pardalos, M.V.F. Pereira, and N. Iliadis, editors, Handbook of Power Systems II, pages 3-30. Springer, 2010.

195. R. A. Jabr. Adjustable robust OPF with renewable energy sources. IEEE Transactions on Power Systems, 28(4):47414751, 2013.

196. R. A. Jabr. Tight polyhedral approximation for mixed-integer linear programming unit commitment formulations. IET Generation, Transmission \& Distribution, 6(11):1104-1111, November 2012.

197. J. Jia and X. Guan. Milp formulation for short-term scheduling of cascaded reservoirs with head effects. In Artificial Intelligence, Management Science and Electronic Commerce (AIMSEC), 2011 2nd International Conference on, pages 4061-4064, 2011.

198. R. Jiang, J. Wang, and Y. Guan. Robust unit commitment with wind power and pumped storage hydro. IEEE Transactions on Power Systems, 27(2):800-810, 2012.

199. R. Jiang, M. Zhang, G. Li, and Y.i Guan. Two-stage robust power grid optimization problem. Preprint : http://www.optimization-online.org/DB_HTML/2010/10/2769.html, pages 1-34, 2010.

200. R.C. Johnson, H.H. Happ, and W.J. Wright. Large scale hydro-thermal unit commitment-method and results. IEEE Transactions on Power Apparatus and Systems, PAS-90(3):1373- 1384, 1971.

201. K.A. Juste, H. Kita, E. Tanaka, and J. Hasegawa. An evolutionary programming solution to the unit commitment problem. IEEE Transactions on Power Systems, 14(4):1452-1459, November 1999.

202. P. Kall and J. Mayer. Stochastic Linear Programming: Models, Theory and Computation. International Series in Operations Research and Management Science. Springer, 1st edition, 2005.

203. J.E. Kelley. The cutting-plane method for solving convex programs. Journal of the Society for Industrial and Applied Mathematics, 8(4):703-712, 1960.

204. R.H. Kerr, J.L. Scheidt, A.J. Fontanna, and J.K. Wiley. Unit commitment. IEEE Transactions on Power Apparatus and Systems, PAS-85(5):417-421, May 1966.

205. A. Keyhani, M.N. Marwali, and M. Dai. Integration of Green and Renewable Energy in Electric Power Systems. Wiley, 1st edition, 2010.

206. K.C. Kiwiel. Bundle methods for convex minimization with partially inexact oracles. To appear in Comp. Opt. Appl, 2012.

207. K. Korad, A.S. Hedman. Robust corrective topology control for system reliability. IEEE Transactions on Power Systems, 28(4):1346-1355, 2013.

208. B.W. Kort and D.P. Bertsekas. A new penalty function method for constrained optimization. In IEEE Conference on Decision and Control, 1972, pages 162-166, 1972.

209. S. Kuloor, G.S. Hope, and O.P. Malik. Environmentally constrained unit commitment. IEE Proceedings C: Generation, Transmission and Distribution, 139(2):122-128, 1992. 
210. R. H. Kwon and D. Frances. Optimization-based bidding in day-ahead electricity auction markets: A review of models for power producers. In A. Sorokin, S. Rebennack, P.M. Pardalos, N.A. Iliadis, and M.V.F. Pereira, editors, Handbook of Networks in Power Systems I, pages 41-60. Springer Heidelberg Berlin, 2012.

211. G Laporte and F.V. Louveaux. The integer l-shaped method for stochastic integer programs with complete recourse. Operations Research Letters, 13(3):133-142, April 1993.

212. G.S. Lauer, N.R. Sandell, D.P. Bertsekas, and T.A. Posbergh. Solution of large-scale optimal unit commitment problems. IEEE Transactions on Power Apparatus and Systems, PAS-101(1):79- 86, 1982.

213. J. Lavaei and S. Low. Zero duality gap in optimal power flow problem. IEEE Transactions on Power Systems, 27(1):92-107, 2012.

214. K.D. Le, R.R. Jackups, J. Feinstein, and J.S. Griffith. Operational aspects of generation cycling. IEEE Transactions on Power Systems, 5(4):1194-1203, May 1990.

215. F.N. Lee. Short-term thermal unit commitment-a new method. IEEE Transactions on Power Systems, 3(2):421428, May 1988.

216. F.N. Lee. The application of commitment utilization factor (CUF) to thermal unit commitment. IEEE Transactions on Power Systems, 6(2):691-698, May 1991.

217. F.N. Lee and Q. Feng. Multi-area unit commitment. IEEE Transactions on Power Systems, 7(2):591- 599, May 1992.

218. F.N Lee, J. Huang, and R. Adapa. Multi-area unit commitment via sequential method and a dc power flow network model. IEEE Transaction on Power Systems, 9(1):297-287, February 1994.

219. C. Lemaréchal. An extension of davidon methods to nondifferentiable problems. Mathematical programming study, 3:95-109, 1975.

220. C. Lemaréchal. Lagrangian relaxation. In [?] (Chapter 4). Springer-Verlag, 2001.

221. C. Lemaréchal, A. Nemirovskii, and Y. Nesterov. New variants of bundle methods. Math. Programming, 69(1):111$147,1995$.

222. C. Lemaréchal and C. Sagastizábal. An approach to variable metric bundle methods. Lecture Notes in Control and Information Science, 197:144-162, 1994.

223. C. Lemaréchal and C. Sagastizábal. Application of bundle methods to the unit-commitment problem. Rapport Technique Nb 0184 INRIA, pages 1-19, 1995.

224. F. Leveque. Competitive Electricity Markets And Sustainability. Edward Elgar Pub, 2002.

225. C. Li, R.B. Johnson, and A.J. Svoboda. A new unit commitment method. IEEE Transaction on Power Systems, 12(1):113-119, February 1997

226. G. Li, J. Lawarree, and C.C. Liu. State-of-the-art of electricity price forecasting in a grid. In S. Rebennack, P.M. Pardalos, M.V.F. Pereira, and N. Iliadis, editors, Handbook of Power Systems II, pages 161-188. Springer-Verlag, 2010 .

227. T. Li and M. Shahidehpour. Strategic bidding of transmission-constrained GENCOs with incomplete information. IEEE Transactions on Power Systems, 20(1):437-447, February 2005.

228. Z. Li and M. Shahidehpour. Generation scheduling with thermal stress constraints. IEEE Transactions on Power Systems, 18(4):1402-1409, 2003.

229. R.-H. Liang and F.-C. Kang. Thermal generating unit commitment using an extended mean field annealing neural network. IEE Proceedings-Generation, Transmission and Distribution, 147(3):164- 170, May 2000.

230. W-M. Lin, F-S. Cheng, and M-T. Tsay. An improved tabu search for economic dispatch with multiple minima. IEEE Transactions on Power Systems, 17(1):108- 112, February 2002.

231. C. Liu, M. Shahidehpour, and L. Wu. Extended benders decomposition for two-stage scuc. IEEE Transactions on Power Systems, 25(2):1192-1194, 2010.

232. C. Liu, J. Wang, and J. Ostrowski. Heuristic prescreening switchable branches in optimal transmission switching. IEEE Transactions on Power Systems, 27(4):2289-2290, 2012.

233. C. Liu, J. Wang, and J. Ostrowski. Static security in multi-period transmission switching. IEEE Transactions on Power Systems, 27(4):1850-1858, 2012.

234. A. Løkketangen and D.L. Woodruff. Progressive hedging and tabu search applied to mixed integer $(0,1)$ multistage stochastic programming. Journal of Heuristics, 2(2):111-128, 1996.

235. F.V. Louveaux and R. Schultz. Stochastic Integer Programming. In [?] (Chapter 4). Elsevier, Amsterdam, 2003.

236. B. Lu and M. Shahidehpour. Unit commitment with flexible generating units. IEEE Transactions on Power Systems, 20(2):1022-1034, May 2005.

237. J-Y. Lucas and T. Triboulet. Hybridization of augmented lagrangian and genetic algorithm for day-to-day unit commitment problem. In META 12 : International Conference on Metaheuristics and Nature Inspired Computing, 2012 .

238. J. Luedtke. A branch-and-cut decomposition algorithm for solving chance-constrained mathematical programs with finite support. Mathematical Programming, To Appear:1-26, 2013.

239. J. Luedtke and S. Ahmed. A sample approximation approach for optimization with probabilistic constraints. SIAM Journal on Optimization, 19:674-699, 2008.

240. D.G. Luenberger and Y. Ye. Linear and Nonlinear Programming, volume 116 of International Series in Operations Research \& Management Science. Springer, 3rd edition, 2010.

241. P.B. Luh, Yajun Wang, and Xing Zhao. Lagrangian relaxation neural network for unit commitment. In IEEE Power Engineering Society 1999 Winter Meeting, volume 1, pages 490-495, 1999.

242. P.B. Luh, D. Zhang, and R.N. Tomastik. An algorithm for solving the dual problem of hydrothermal scheduling. IEEE Transactions on Power Systems, 13:593600, 1998.

243. M. Di Lullo. Modelli di ottimizzazione per lo unit commitment con optimal transmission switching: Analisi e implementazione. Master's thesis, Facoltá di Ingegneria dell'Informazione, Informatica e Statistica, Universitá di Roma La Sapienza, Piazzale Aldo Moro, 5 00185, Roma, 2013. 
244. M. Madrigal and V.H. Quintana. An interior-point/cutting-plane method to solve unit commitment problems. IEEE Transactions on Power Systems, 15(3):1022-1027, 2000.

245. S. Makkonen and R. Lahdelma. Non-convex power plant modelling in energy optimisation. European Journal of Operational Research, 171:1113-1126, 2006.

246. A.H. Mantawy, Y.L. Abdel-Magid, and S.Z. Selim. A simulated annealing algorithm for unit commitment. IEEE Transactions on Power Systems, 13(1):197- 204, February 1998.

247. A.H. Mantawy, S.A. Soliman, and M.E. El-Hawary. A new tabu search algorithm for the long-term hydro scheduling problem. In LESCOPE 02 Large Engineering Systems Conference on Power Engineering 2002, pages 29-34, 2002.

248. A. Merlin and P. Sandrin. A new method for unit commitment at Electricité de France. IEEE Transactions Power App. Syst., PAS-102:1218-1225, 1983.

249. A.J. Mezger and K.C. de Almeida. Short term hydrothermal scheduling with bilateral transactions via bundle method. Electrical Power and Energy Systems, 29:387-396, 2007.

250. M. Minoux. Solving some multistage robust decision problems with huge implicitly defined scenario trees. Algorithmic Operations Research, 4(1):1-18, 2009

251. M. Minoux. Two-stage robust optimization, state-space representable uncertainty and applications. $R A I R O$ Operations Research, 48:455-475, 2014

252. J. Miranda, A. Wanga, R. Botterud, H. Bessa, L. Keko, D. Carvalho, J. Issicaba, and V. Sumaili. Wind power forecasting uncertainty and unit commitment. Applied Energy, 88:4014-4023, 2011.

253. S. Mokhtari, J. Sing, and B. Wollenberg. A unit commitment expert system. IEEE Transactions on Power Systems, 3(1):272- 277, February 1988.

254. D. K. Molzahn, J. T. Holzer, B. C. Lesieutre, and C. L. DeMarco. Implementation of a large-scale optimal power flow solver based on semidefinite programming. IEEE Transactions on Power Systems, 28(4):3987-3998, 2013.

255. J. A. Momoh, R. Adapa, and M. E. El-Hawary. A review of selected optimal power flow literature to 1993. i. nonlinear and quadratic programming approaches. IEEE Transactions on Power Systems, 14:96-104, 1999.

256. J. A. Momoh, R. Adapa, and M. E. El-Hawary. A review of selected optimal power flow literature to 1993. ii. newton, linear programming and interior point methods. IEEE Transactions on Power Systems, 14:105-111, 1999.

257. G. Morales-España, J.M. Latorre, and A. Ramos. Tight and compact MILP formulation for the thermal unit commitment problem. IEEE Transactions on Power Systems, 28(4):4897-4908, 2013.

258. G. Morales-España, J.M. Latorre, and A. Ramos. Tight and compact MILP formulation of start-up and shut-down ramping in unit commitment. IEEE Transactions on Power Systems, 28(2):1288-1296, 2013.

259. G. Morales-España, A. Ramos, and J. García-González. An MIP formulation for joint market-clearing of energy and reserves including ramp scheduling. IEEE Transactions on Power Systems, to appear, 2014.

260. H. Mori and O. Matsuzaki. Embedding the priority list into tabu search for unit commitment. In IEEE Power Engineering Society Winter Meeting, 2001, volume 3, pages 1067-1072, 2001.

261. P. S. Moura and A.T. de Almeida. Large scale integration of wind power generation. In S. Rebennack, P.M. Pardalos, M.V.F. Pereira, and N. Iliadis, editors, Handbook of Power Systems I, pages 95-120. Springer-Verlag, 2010.

262. J.A. Muckstadt and S.A. Koenig. An application of lagrangian relaxation to scheduling in power-generation systems Operations Research, 25(3):387-403, June 1977.

263. J.A. Muckstadt and R.C. Wilson. An application of mixed-integer programming duality to scheduling thermal generating systems. IEEE Transactions on Power Apparatus and Systems, PAS-87(12):1968-1978, December 1968.

264. A. Muñoz, E. F. Sánchez Úbeda, A. Cruz, and J. Marín. Short-term forecasting in power systems: A guided tour. In S. Rebennack, P.M. Pardalos, M.V.F. Pereira, and N. Iliadis, editors, Handbook of Power Systems II, pages 129-160. Springer-Verlag, 2010

265. C. Murillo-Sanchez and R.J. Thomas. Thermal unit commitment including optimal ac power flow constraints. In Thirty-First Hawaii International Conference on System Sciences, volume 3, 1998.

266. R. Nayak and J.D. Sharma. A hybrid neural network and simulated annealing approach to the unit commitment problem. Computers and Electrical Engineering, 26(6):461-477, 2000.

267. A. Nemirovski and A. Shapiro. Scenario approximations of chance constraints. Preprint : http://www.optimizationonline.org/DB_HTML/2004/11/1000.html, pages 1-45, 2004.

268. A. Nemirovski and A. Shapiro. Convex approximations of chance constrained programs. SIAM Journal of Optimization, 17(4):969-996, 2006.

269. A. Nemirovski and A. Shapiro. Scenario Approximations of Chance Constraints (Chapter 1 in [?]). Springer, 2006.

270. Y. Nesterov. Primal-dual subgradient methods for convex problems. Mathematical Programming, 120(1):221-259 2009.

271. A. Nguyen-Huu. Valorisation financière sur les marchés d'électricité. PhD thesis, Paris Dauphine, July 2012.

272. E. Ni, X. Guan, and R. Li. Scheduling hydrothermal power systems with cascaded and head-dependent reservoirs. IEEE Transactions on Power Systems, 14:1127-1132, 1999.

273. E. Ni, P. B. Luh, and S. Rourke. Optimal integrated generation bidding and scheduling with risk management under a deregulated power market. IEEE Transactions on Power Systems, 19(1):600-609, 2004.

274. O. Nilsson and D. Sjelvgren. Mixed-integer programming applied to short-term planning of a hydro-thermal system. IEEE Transactions on Power Systems, 11(1):281-286, February 1996.

275. F.J. Nogales, J. Contreras, A.J. Conejo, and R. Espínola. Forecasting next-day electricity prices by time series models. IEEE Transactions on Power Systems, 17(2):342-348, May 2002.

276. M.P. Nowak. Stochastic Lagrangian relaxation in power scheduling of a hydrothermal system under uncertainty. PhD thesis, Humboldt University Berlin, 2000.

277. M.P. Nowak and W. Römisch. Stochastic lagrangian relaxation applied to power scheduling in a hydro-thermal system under uncertainty. Annals Of Operations Research, 100(1-4):251-272, December 2000.

278. R. Nürnberg and W. Römisch. A two-stage planning model for power scheduling in a hydro-thermal system under uncertainty. Optimization and Engineering, 3:355-378, December 2003. 
279. A. R. L. Oliveira, S. Soares, and L. Nepomuceno. Short term hydroelectric scheduling combining network flow and interior point approaches. Electrical Power and Energy Systems, 27:91-99, 2005.

280. R.P. O'Neill, K.W. Hedman, E.A. Krall, A. Papavasiliou, and S.S. Oren. Economic analysis of the n-1 reliable unit commitment and transmission switching problem using duality concepts. Energy Systems, 1(2):165-195, 2010.

281. S.S. Oren, A.J. Svoboda, and R.B. Johnson. Volatility of unit commitment in competitive electricity markets. In International Conference on System Sciences, volume 5, pages 594-601, 1997.

282. J. Ostrowski, M.F. Anjos, and A. Vannelli. Tight mixed integer linear programming formulations for the unit commitment problem. IEEE Transactions on Power Systems, 27(1):39-46, February 2012.

283. J. Ostrowski, A. Vannelli, M.F. Anjos, and Québec) Groupe d'études et de recherche en analyse des décisions (Montréal. Symmetry in Scheduling Problems. Groupe d'études et de recherche en analyse des décisions, 2010.

284. J. Ostrowski and J. Wang. Network reduction in the transmission-constrained unit commitment problem. Computers E Industrial Engineering, 63(1):702-707, November 2012.

285. J. Ostrowski, J. Wang, and C. Liu. Exploiting symmetry in transmission lines for transmission switching. IEEE Transactions on Power Systems, 27(3):1708-1709, 2012.

286. N. Oudjane, J. Collet, and V. Duwig. Some non-gaussian models for electricity spot prices. 9th International Conference on Probabilistic Methods Applied to Power Systems, 2006.

287. Z. Ouyang and M. Shahidehpour. An intelligent dynamic programming for unit commitment application. IEEE Transactions on Power Systems, 6(3):1203- 1209, August 1991.

288. Z. Ouyang and M. Shahidehpour. A hybrid artificial neural network-dynamic programming approach to unit commitment. IEEE Transactions on Power Systems, 7(1):236- 242, February 1992.

289. U.A. Ozturk, M. Mazumdar, and B.A. Norman. A solution to the stochastic unit commitment problem using chance constrained programming. IEEE Transactions on Power Systems, 19(3):1589-1598, August 2004.

290. N. P. Padhy. Unit commitment - a bibliographical survey. IEEE Transaction On Power Systems, 19(2):1196-1205, May 2004.

291. S. I. Palamarchuk. Compromise scheduling of bilateral contracts in electricity market environment. In A. Sorokin, S. Rebennack, P.M. Pardalos, N.A. Iliadis, and M.V.F. Pereira, editors, Handbook of Networks in Power Systems I, pages 241-262. Springer Heidelberg Berlin, 2012.

292. C.K. Pang and H.C. Chen. Optimal short-term thermal unit commitment. IEEE Transactions on Power Apparatus and Systems, 95(4):1336- 1346, July 1976.

293. C.K. Pang, G.B. Sheble, and F. Albuyeh. Evaluation of dynamic programming based methods and multiple area representation for thermal unit commitments. IEEE Transactions on Power Apparatus and Systems, PAS-100(3):12121218, March 1981.

294. A. Papavasiliou and S. S. Oren. A stochastic unit commitment model for integrating renewable supply and demand response. In Invited Panel Paper, Proceeding of the IEEE PES GM, San Diego, CA, July 24-28, 2012, 2012.

295. A. Papavasiliou and S. S. Oren. A comparative study of stochastic unit commitment and security-constrained unit commitment using high performance computing. In Proceeding of the European Control Conference ECC 2013, 2013.

296. A. Papavasiliou, S. S. Oren, and R. O'Neill. Reserve requirements for wind power integration: A scenario-based stochastic programming framework. IEEE Transactions on Power Systems, 26(4):2197-2206, 2011.

297. A. Papavasiliou, S. S. Oren, and R. O'Neill. Multi-area stochastic unit commitment for high wind penetration in a transmission constrained network. Operations Research, 61(3):578-592, 2013.

298. A. Papavasiliou, S. S. Oren, Z. Yang, P. Balasubramanian, and K. W. Hedman. An application of high performance computing to transmission switching. In IREP Bulk Power System Dynamics and Control Symposium, Rethymnon, Greece, 2013, 2013

299. E. Parrilla and J. García-González. Improving the B\&B search for large-scale hydrothermal weekly scheduling problems. Electrical Power and Energy Systems, 28:339-348, 2006.

300. D.J. Pedregal, J. Contreras, and A. A. Sanchez de la Nieta. Ecotool: A general matlab forecasting toolbox with applications to electricity markets. In A. Sorokin, S. Rebennack, P.M. Pardalos, N.A. Iliadis, and M.V.F. Pereira, editors, Handbook of Networks in Power Systems I, pages 151-171. Springer Heidelberg Berlin, 2012.

301. T. Peng and K. Tomsovic. Congestion influence on bidding strategies in an electricity market. IEEE Transactions on Power Systems, 18(3):1054-1061, August 2003.

302. W. Pepper, B.J. Ring, E.G. Read, and S.R. Starkey. Short-term electricity market prices: A review of characteristics and forecasting methods. In A. Sorokin, S. Rebennack, P.M. Pardalos, N.A. Iliadis, and M.V.F. Pereira, editors, Handbook of Networks in Power Systems II, pages 3-36. Springer Heidelberg Berlin, 2012.

303. M.V. Pereira, S. Granville, M.H.C. Fampa, R. Dix, and L.A. Barroso. Strategic bidding under uncertainty: A binary expansion approach. IEEE Transactions on Power Systems, 11(1):180-188, February 2005.

304. M.V.F. Pereira and L.M.V.G. Pinto. Application of decomposition techniques to the mid - and short - term scheduling of hydrothermal systems. IEEE Transactions on Power Apparatus and Systems, PAS-102(11):3611-3618, November 1983.

305. A. Philpott and R. Schultz. Unit commitment in electricity pool markets. Mathematical Programming: Series B, 108:313-337, 2006.

306. M. Piekutowki, T. Litwinowcz, and R. Frowd. Optimal short-term scheduling for a large-scale cascaded hydro system. IEEE Transactions on Power Systems, 9(2):805-811, May 1994.

307. P.O. Pineau and P. Murto. An oligopolistic investment model of the finnish electricity market. Annals of Operations Research, 121(1-4):123-148, July 2003.

308. B.T. Polyak. Subgradient methods: a survey of soviet research. In C. Lemaréchal and R. Mifflin, editors, Nonsmooth Optimization, IIASA Proceedings Series. Pergamon Press, 1977.

309. A. Prékopa. Stochastic Programming. Kluwer, Dordrecht, 1995.

310. A. Prékopa. Probabilistic programming. In [?] (Chapter 5). Elsevier, Amsterdam, 2003. 
311. A. Prékopa, T. Rapcsák, and I. Zsuffa. Serially linked reservoir system design using stochastic programming. Water Resources Research, 14:672678, 1978

312. J.E. Price. Market-based price differentials in zonal and lmp market designs. IEEE Transaction on Power Systems, 22(4):1486-1494, November 2007.

313. C.C.A. Rajan and M.R. Mohan. An evolutionary programming-based tabu search method for solving the unit commitment problem. IEEE Transactions on Power Systems, 19(1):577- 585, February 2004.

314. C.C.A. Rajan, M.R. Mohan, and K. Manivannan. Neural-based tabu search method for solving unit commitment problem. IEEE Proceedings-Generation, Transmission and Distribution, 150(4):469-474, July 2003.

315. C.C.A. Rajan, S.C. Selvi, and R. P. Kumudini Devi. Multi-area unit commitment with transmission losses using evolutionary iteration particle swarm optimization approach. European Journal of Scientific Research, 76(4):672$691,2012$.

316. A. Ramos, S. Cerisola, J. M. Latorre, R. Bellido, A. Perea, and E. Lopez. A decision support model forweekly operation of hydrothermal systems by stochastic nonlinear optimization. In M. Bertocchi, G. Consigli, and M. A.H. Dempster, editors, Stochastic Optimization Methods in Finance and Energy: New Financial Products and Energy Market Strategies, pages 143-162. Springer, 2012.

317. M. Razaviyayn, M. Hong, and Z-Q. Luo. A unified convergence analysis of block successive minimization methods for nonsmooth optimization. Technical report, University of Minnesota Twin Cites, 2012.

318. E.G. Read. Co-optimization of energy and ancillary service markets. In S. Rebennack, P.M. Pardalos, M.V.F. Pereira, and N. Iliadis, editors, Handbook of Power Systems I, pages 307-330. Springer-Verlag, 2010.

319. N.J. Redondo and A.J. Conejo. Short-term hydro-thermal coordination by lagrangian relaxation: solution of the dual problem. IEEE Transactions on Power Systems, 14:8995, 1999.

320. P. Rocha and T. K. Das. Finding joint bidding strategies for day-ahead electricity and related markets. In A. Sorokin, S. Rebennack, P.M. Pardalos, N.A. Iliadis, and M.V.F. Pereira, editors, Handbook of Networks in Power Systems I, pages 61-88. Springer Heidelberg Berlin, 2012.

321. R. T. Rockafellar and Roger J.-B. Wets. Scenarios and policy aggregation in optimization under uncertainty. Mathematics of Operations Research, 16(1):119-147, February 1991.

322. W. Römisch. Stability of Stochastic Programming Problems. In [?] (Chapter 8). Elsevier, Amsterdam, 2003.

323. W. Römisch and R. Schultz. Distribution sensitivity for certain classes of chance-constrained models with application to power dispatch. Journal of Optimization Theory and Applications, 71:569-588, 1991.

324. W. Römisch and R. Schultz. Decomposition of a multi-stage stochastic program for power dispatch. SUPPL, 3:29-32, 1996.

325. W. Römisch and S. Vigerske. Recent progress in two-stage mixed-integer stochastic programming with applications to power production planning. In S. Rebennack, P.M. Pardalos, M.V.F. Pereira, and N. Iliadis, editors, Handbook of Power Systems I, pages 177-208. Springer-Verlag, 2010.

326. P. A. Ruiz, C. R. Philbrick, E. J. Zak, K. W. Cheung, and P. W. Sauer. Uncertainty management in the unit commitment problem. IEEE Transactions on Power Systems, 24(2):642- 651, May 2009.

327. P. A. Ruiz, A. Rudkevich, M. C. Caramanis, E. Goldis, E. Ntakou, and C. R. Philbrick. Reduced MIP formulation for transmission topology control. In Allerton Conference, 2012, pages 1073-1079. IEEE, 2012.

328. A. Ruszczyński. On convergence of an augmented lagrangian decomposition method for sparse convex optimization. Mathematics of Operations Research, 20(3):634-656, 1995.

329. A. Ruszczyński. Decomposition Methods (Chapter 3 in [?]), volume 10 of Handbooks in Operations Research and Management Science. Elsevier, Amsterdam, 2003.

330. A. Ruszczyński and A. Shapiro. Multi-Stage problems. Chapter 3 in [?]. MPS-SIAM series on optimization. SIAM and MPS, Philadelphia, 2009.

331. A. Ruszczyński and A. Shapiro. Two Stage problems. Chapter 2 in [?]. MPS-SIAM series on optimization. SIAM and MPS, Philadelphia, 2009.

332. S. Ruzic and R. Rajakovic. Optimal distance method for lagrangian multipliers updating in short-term hydro-thermal coordination. IEEE Transactions on Power Systems, 13:1439-1444, 1998.

333. C. Sagastizábal. Divide to conquer: Decomposition methods for energy optimization. Mathematical Programming, 134(1):187-222, 2012.

334. M.S. Salam, A.R. Hamdan, and K.M. Nor. Integrating an expert system into a thermal unit-commitment algorithm. IEE Proceedings Generation, Transmission and Distribution, 138(6):553-559, November 1991.

335. S. Salam, K.M. Nor, and A.R. Hamdan. Comprehensive algorithm for hydrothermal coordination. IEE Transactions on Generation Transmission and Distribution, 144:482-488, 1997.

336. S. Salam, K.M. Nor, and A.R. Hamdan. Hydrothermal scheduling based lagrangian relaxation approach to hydrothermal coordination. IEEE Transactions on Power Systems, 13:226-235, 1998.

337. B. Saravanan, S. Das, S. Sikri, and D.P. Kothari. A solution to the unit commitment problem: a review. Front. Energy, 7(2):223-236, 2013.

338. A.T. Sarić and A.M. Stankovic. Finitely adaptive linear programming in robust power system optimization. In Power Tech, 2007 IEEE Lausanne, pages 1302-1307, 2007.

339. H. Sasaki, M. Watanabe, J. Kubokawa, N. Yorino, and R. Yokoyama. A solution method of unit commitment by artificial neural networks. IEEE Transactions on Power Systems, 7(3):974- 981, August 1992.

340. E. Sauma, S. Jerardino, C. Barria, R. Marambio, A. Brugman, and J. Mejia. Electric interconnections in the andes community: Threats and opportunities. In A. Sorokin, S. Rebennack, P.M. Pardalos, N.A. Iliadis, and M.V.F. Pereira, editors, Handbook of Networks in Power Systems I, pages 345-366. Springer Heidelberg Berlin, 2012.

341. M.A.H. El Sayed. Solar supported steam production for power generation in Egypt. Energy Policy, 33(10):1251-1259, July 2005. 
342. R. Schultz, M. Nowak, R. Nürnberg, W. Römisch, and M. Westphalen. Stochastic programming for power production and trading under uncertainty. In W. Jvsger and H.-J. Krebs, editors, Mathematics - Key Technology for the Future, pages 623-636. Springer Berlin Heidelberg, September 2003.

343. M.H. Sendaula, S.K. Biswas, A. Eltom, C. Parten, and W. Kazibwe. Application of artificial neural networks to unit commitment. In Proceedings of the First International Forum on Applications of Neural Networks to Power Systems, 1991, pages 256-260, 1991.

344. S. Senthil-Kumar and V. Palanisamy. A dynamic programming based fast computation hopfield neural network for unit commitment and economic dispatch. Electric Power Systems Research, 77(8):917-925, June 2007.

345. M. Shafie-Khah, M. Parsa Moghaddam, and M.K. Sheikh-El-Eslami. Unified solution of a non-convex scuc problem using combination of modified branch-and-bound method with quadratic programming. Energy Conversion and Management, 52(12):3425-3432, December 2011.

346. M. Shahidehpour, H. Yamin, and Z. Li. Market Operations in Electric Power Systems: Forecasting, Scheduling, and Risk Management. Wiley-IEEE Press, 2002.

347. T.A.M. Sharaf and G.J. Berg. Voltampere reactive compensation using chance-constrained programming. IEEE Proceedings C Generation, Transmission and Distribution, 129(1):24- 29, January 1982.

348. J.J. Shaw, R.F. Gendron, and D.P. Bertsekas. Optimal scheduling of large hydrothermal power systems. IEEE Power Engineering Review, PER-5(2):32, February 1985.

349. G.B. Sheble and G.N. Fahd. Unit commitment literature synopsis. IEEE Transactions onPower Systems, 9(1):128135, Feb 1994.

350. G.B. Sheble, T.T. Maifeld, K. Brittig, G. Fahd, and S. Fukurozaki-Coppinger. Unit commitment by genetic algorithm with penalty methods and a comparison of lagrangian search and genetic algorithmeconomic dispatch example. International Journal of Electrical Power E Energy Systems, 18(6):339-346, August 1996.

351. H.D. Sherali and W.P. Adams. A Reformulation-Linearization Technique for Solving Discrete and Continuous Nonconvex Problems. Nonconvex Optimization and Its Applications. Springer, 1998.

352. H.D. Sherali and B.M.P. Fraticelli. A modification of benders' decomposition algorithm for discrete subproblems: an approach for stochastic programs with integer recourse. Journal of Global Optimization, 22:319-342, 2002.

353. T. Shiina. Numerical solution technique for joint chance-constrained programming problem "an application to electric power capacity expansion". Journal of the Operations Research Society of Japan, 42(2):128-140, June 1999.

354. T. Shiina and J.R. Birge. Stochastic unit commitment problem. International Transactions in Operational Research, 11(1):19-32, January 2004.

355. H. Siahkali and M. Vakilian. Stochastic unit commitment of wind farms integrated in power system. Electric Power Systems Research, 80(9):1006-1017, September 2010.

356. W. Sifuentes and A. Vargas. Hydrothermal scheduling using benders decomposition: accelerating techniques. IEEE Transactions on Power Systems, 22:1351-1359, 2007.

357. W. Sifuentes and A. Vargas. Short-term hydrothermal coordination considering an ac network modeling. International Journal of Electrical Power ES Energy Systems, 29:488-496, 2007.

358. D. N. Simopoulos, S. D. Kavatza, and C. D. Vournas. Unit commitment by an enhanced. IEEE Transactions on Power Systems, 21(1):68- 76, February 2006.

359. P.K. Singhal and R.N. Sharma. Dynamic programming approach for large scale unit commitment problem. In International Conference on Communication Systems and Network Technologies (CSNT), 2011, pages 714-717, 2011.

360. T.K. Siu, G.A. Nash, and Z.K. Shawwash. A practical hydro, dynamic unit commitment and loading model. IEEE Transactions on Power Systems, 16(2):301- 306, May 2001.

361. W.L. Snyder, H.D. Powell, and J.C. Rayburn. Dynamic programming approach to unit commitment. IEEE Transactions on Power Systems, 2(2):339 - 348, May 1987.

362. A. Street, F. Oliveira, and J.M. Arroya. Contingency-constrained unit commitment with $n-k$ security criterion: A robust optimization approach. IEEE Transactions on Power Systems, 26(3):1581-1590, 2011.

363. M. Sudhakaran and P. Ajay-D-Vimal Raj. Integrating genetic algorithms and tabu search for unit commitment. International Journal of Engineering, Science and Technology, 2(1):57-69, 2010.

364. F. Y. K. Takigawa, E. L. da Silva, E. C. Finardi, and R. N. Rodrigues. Solving the hydrothermal scheduling problem considering network constraints. Electric Power Systems Research, 88:89-97, 2012.

365. F.Y.K. Takigawa, E.C. Finardi, and E.L. da Silva. A decomposition strategy to solve the short-term hydrothermal scheduling based on lagrangian relaxation. Journal of Algorithms and Optimization, 1(1):13-24, 2013.

366. S. Takriti and J.R. Birge. Using integer programming to refine lagrangian-based unit commitment solutions. IEEE Transactions on Power Systems, 15(1):151-156, 2000.

367. S. Takriti, J.R. Birge, and E. Long. A stochastic model for the unit commitment problem. IEEE Transactions on Power Systems, 11:1497-1508, 1996.

368. S. Takriti, B. Krasenbrink, and L.S.Y. Wu. Incorporating fuel constraints and electricity spot prices into the stochastic unit commitment problem. Operations Research, 48(2):268-280, April 2000.

369. S.K. Tong and M. Shahidehpour. Combination of lagrangian-relaxation and linear-programming approaches for fuelconstrained unit-commitment problems. IEEE Proceedings Generation, Transmission and Distribution, 136(3):162174, May 1989.

370. C. Triki, P. Beraldi, and G. Gross. Optimal capacity allocation in multi-auction electricity markets under uncertainty. Computers \& Operations Research, 32:201-217, 2005.

371. C. Triki, A. J. Conejo, and L.P. Garcés. Short-term trading for electricity producers. In M. Bertocchi, G. Consigli, and M.A.H. Dempster, editors, Stochastic Optimization Methods in Finance and Energy: New Financial Products and Energy Market Strategies, volume 163 of International Series in Operations Research ES Management Science, pages 181-202. Springer-Verlag, 2011. 
372. S. Trukhanova, L. Ntaimo, and A. Schaefer. Adaptive multicut aggregation for two-stage stochastic linear programs with recourse. European Journal of Operational Research, 206(2):395-406, October 2010.

373. C.L. Tseng, C.A. Li, and S.S. Oren. Solving the unit commitment problem by a unit decommitment method. Journal of Optimization Theory and Applications, 105(3):707-730, June 2000.

374. P. Tseng. Convergence of a block coordinate descent method for nondifferentiable minimization. Journal of Optimization Theory and Applications, 109(3):475-494, 2001.

375. A. Tuohy, P. Meibom, E. Denny, and M.J. O'Malley. Unit commitment for systems with significant wind penetration. IEEE Transactions on Power Systems, 24(2):592-601, May 2009.

376. A. Turgeon. Optimal scheduling of thermal generating units. IEEE Transactions on Automatic Control, 23(6):10001005, December 1978 .

377. J. Valenzuela and M. Mazumdar. Commitment of electric power generators under stochastic market prices. Operations Research, 51(6):880-893, 2003.

378. J. Valenzuela and A. E. Smith. A seeded memetic algorithm for large unit commitment problems. Journal of Heuristics, 8(2):173-195, March 2002.

379. W. van Ackooij. Decomposition approaches for block-structured chance-constrained programs with application to hydro-thermal unit commitment. To Appear in Mathematical Methods of Operations Research, pages 1-21, 2014.

380. W. van Ackooij, R. Henrion, A. Möller, and R. Zorgati. On probabilistic constraints induced by rectangular sets and multivariate normal distributions. Mathematical Methods of Operations Research, 71(3):535-549, 2010.

381. W. van Ackooij, R. Henrion, A. Möller, and R. Zorgati. Chance Constrained Programming and Its Applications to Energy Management. In [?] (Chapter 13). INTECH, 2011.

382. W. van Ackooij, R. Henrion, A. Möller, and R. Zorgati. Joint chance constrained programming for hydro reservoir management. Optimization and Engineering, 15:509-531, 2014.

383. W. van Ackooij and J. Malick. Decomposition algorithm for large-scale two-stage unit-commitment. draft submitted, pages 1-26, 2014.

384. W. van Ackooij and J. Wirth. Un jeu d'acteurs n-zones pour SSPS. synthèse et propositions. Technical Report H-R33-2006-03913-FR, EDF R\&D, 2007.

385. R.M. van Slyke and R.J-B. Wets. L-shaped linear programs with applications to optimal control and stochastic programming. SIAM Journal of Applied Mathematics, 17:638-663, 1969.

386. M. Ventosa, A. Baíllo, A. Ramos, and M. Rivier. Electricity market modeling trends. Energy Policy, 33(7):897-913, 2005.

387. T.A.A. Victoire and A.E. Jeyakumar. Unit commitment by a tabu-search-based hybrid-optimisation technique. IEE Proceedings-Generation, Transmission and Distribution, 152(4):563- 574, July 2005.

388. J.C. Villumsen and A.B. Philpott. Column generation for transmission switching of electricity networks with unit commitment. Proceedings of the International MultiConference of Engineers and Computer Scientists, $2,2011$.

389. S. Vucetic, K. Tomsovic, and Z. Obradovic. Discovering price-load relationships in California's electricity market. IEEE Transactions on Power Systems, 16(2):280-286, May 2001.

390. S.W. Wallace and S.-E. Fleten. Stochastic programming models in energy (chapter 10 in [?]). In A. Ruszczynski and A. Shapiro, editors, Stochastic Programming, volume 10 of Handbooks in Operations Research and Management Science, pages 637-677. Elsevier, 2003.

391. M.P. Walsh and M.J. O'Malley. Augmented hopfield network for unit commitment and economic dispatch. IEEE Transactions on Power Systems, 12(4):1765- 1774, November 1997.

392. C. Wang and M. Shahidehpour. Effects of ramp-rate limits on unit commitment and economic dispatch. IEEE Transactions on Power Systems, 8(3):1341- 1350, August 1993.

393. J. Wang, M. Shahidehpour, and Z. Li. Security-constrained unit commitment with volatile wind power generation. IEEE Transactions on Power Systems, 23(3):1319-1327, 2008.

394. J. Wang, J. Wang, C. Liu, and J. P. Ruiz. Stochastic unit commitment with sub-hourly dispatch constraints. Applied Energy, 105:418422, 2013.

395. J. Wang, X. Wang, and Y. Wu. Operating reserve model in the power market. IEEE Transactions on Power Systems, 20(1):223-229, February 2005

396. L. Wang, M. Mazumdar, M.D. Bailey, and J. Valenzuela. Oligopoly models for market price of electricity under demand uncertainty and unit reliability. European Journal of Operational Research, 181(3):1309-1321, September 2007 .

397. Q. Wang, Y. Guan, and J. Wang. A chance-constrained two-stage stochastic program for unit commitment with uncertain wind power output. IEEE Transactions on Power Systems, 27(1):206-215, 2012.

398. Q. Wang, J-P. Watson, and Y. Guan. Two-stage robust optimization for $n-k$ contingency-constrained unit commitment. IEEE Transactions on Power Systems, 28(3):2366-2375, 2013

399. S.J. Wang, M. Shahidehpour, D.S. Kirschen, S. Mokhtari, and G.D. Irisarri. Short-term generation scheduling with transmission and environmental constraints using an augmented lagrangian relaxation. IEEE Transactions on Power Systems, 10(3):1294-1301, August 1995

400. Y. Wang, Q. Xia, and C. Kang. Unit commitment with volatile node injections by using interval optimization. IEEE Transactions on Power Systems, 26(3):1705-1713, August 2011.

401. F. Wen and A.K. David. Optimal bidding strategies and modeling of imperfect information among competitive generators. IEEE Transactions on Power Systems, 16(1):15-21, February 2001

402. P. Wolfe. A method of conjugate subgradients for minimizing nondifferentiable functions. Mathematical programming study, 3:143-173, 1975 .

403. K.P. Wong and Y.W. Wong. Genetic and genetic/simulated-annealing approaches to economic dispatch. IEEE Proceedings-Generation, Transmission and Distribution, 141(5):507- 513, September 1994. 
404. K.P. Wong and Y.W. Wong. Combined genetic algorithm/simulated annealing/fuzzy set approach to short-term generation scheduling with take-or-pay fuel contract. IEEE Transactions on Power Systems, 11(1):128-136, February 1996.

405. S. Wong and J.D. Fuller. Pricing energy and reserves using stochastic optimization in an alternative electricity market. IEEE Transactions on Power Systems, 22(2):631- 638, May 2007.

406. A.J. Wood and B.F. Wollemberg. Power Generation Operation and Control. John Wiley \& Sons, 1996.

407. L. Wu. An improved decomposition framework for accelerating LSF and BD based methods for network-constrained UC problems. IEEE Transactions on Power Systems, 28(4):3977-3986, 2013.

408. L. Wu. A tighter piecewise linear approximation of quadratic cost curves for unit commitment problems. IEEE Transactions on Power Systems, 26(4):2581-2583, November 2011.

409. L. Wu, M. Shahidehpour, and T. Li. Stochastic security-constrained unit commitment. IEEE Transactions on Power Systems, 22(2), May 2007.

410. L. Wu, M. Shahidehpour, and Z. Li. Comparison of scenario-based and interval optimization approaches to stochastic SCUC. IEEE Transactions on Power Systems, 27(2):913-921, May 2012.

411. L. Wu, M. Shahidehpour, and L. Tao. Stochastic security-constrained unit commitment. IEEE Transactions on Power Systems, 22(2):800- 811, May 2007.

412. P. Xiong and P. Jirutitijaroen. Stochastic unit commitment using multi-cut decomposition algorithm with partial aggregation. In IEEE Power and Energy Society General Meeting, 2011.

413. H. Yan, P.B. Luh, X. Guan, and P.M. Rogan. Scheduling of hydro-thermal power systems. IEEE Transactions on Power Systems, 8(3):1358-1365, August 1993.

414. H. Yan, P.B. Luh, and L. Zhang. Scheduling of hydrothermal power systems using the augmented lagrangian decomposition and coordination technique. In American Control Conference 1994, volume 2, pages 1558-1562, August 1994.

415. H.T. Yang, P.C. Yang, and C.L. Huang. Evolutionary programming based economic dispatch for units with nonsmooth fuel cost functions. IEEE Transactions on Power Systems, 11(1):112- 118, February 1996.

416. J-S. Yang and N. Chen. Short term hydrothermal coordination using multi-pass dynamic programming. IEEE Transactions on Power Systems, 4(3):1050-1056, 1989.

417. Z. Yu, F.T. Sparrow, B. Bowen, and F.J. Smardo. On convexity issues of short-term hydrothermal scheduling. Electrical Power and Energy Systems, 20:451-457, 2000.

418. S. Zaourar and J. Malick. Prices stabilization for inexact unit-commitment problems. Mathematical Methods of Operations Research, to Appear, pages 1-19, 2013.

419. H. Zareipour. Short-term electricity market prices: A review of characteristics and forecasting methods. In A. Sorokin, S. Rebennack, P.M. Pardalos, N.A. Iliadis, and M.V.F. Pereira, editors, Handbook of Networks in Power Systems I, pages 89-121. Springer Heidelberg Berlin, 2012.

420. C. Zhang and J. Wang. Optimal transmission switching considering probabilistic reliability. IEEE Transactions on Power Systems, 2014. to appear.

421. D. Zhang, P.B. Luh, and Y. Zhang. A bundle method for hydrothermal scheduling. IEEE Transactions on Power Systems, 14:13551361, 1999.

422. C. Zhao and Y. Guan. Unified stochastic and robust unit commitment. IEEE Transactions on Power Systems, 28(3):3353-3361, 2013.

423. C. Zhao, J. Wang, J-P. Watson, and Y. Guan. Multi-stage robust unit commitment considering wind and demand response uncertainties. IEEE Transactions on Power Systems, 28(3):2708-2717, 2013.

424. L. Zhao and B. Zeng. Robust unit commitment problem with demand response and wind energy. In Proceedings of IEEE Power and Energy Society General Meeting, 2012, 2012.

425. Q. Zheng, J. Wang, P. Pardalos, and Y. Guan. A decomposition approach to the two-stage stochastic unit commitment problem. Annals of Operations Research, 210(1):387-410, 2013.

426. J. Zhu. Optimization of Power System Operation. IEEE Press Series on Power Engineering. Wiley-IEEE Press, 2009.

427. F. Zhuang and F.D. Galiana. Towards a more rigorous and practical unit commitment by lagrangian relaxation. IEEE Transactions on Power Systems, 3(2):763-773, May 1988.

428. F. Zhuang and F.D. Galiana. Unit commitment by simulated annealing. IEEE Transactions on Power Systems, 5(1):311-318, Febraury 1990.

429. R. Zorgati and W. van Ackooij. Optimizing financial and physical assets with chance-constrained programming in the electrical industry. Optimization and Engineering, 12(1):237-255, 2011. 